\title{
Digital image correlation for sensing kinematic fields in manufacturing processes: a review
}

\author{
Pingan Zhu and Chao Zhang \\ Zhejiang University, Hangzhou, China, and \\ Jun Zou \\ Mechanical Engineering, Zhejiang University, Hangzhou, China
}

\begin{abstract}
Purpose - The purpose of the work is to provide a comprehensive review of the digital image correlation (DIC) technique for those who are interested in performing the DIC technique in the area of manufacturing. Design/methodology/approach - No methodology was used because the paper is a review article. Findings - no fundings.

Originality/value - Herein, the historical development, main strengths and measurement setup of DIC are introduced. Subsequently, the basic principles of the DIC technique are outlined in detail. The analysis of measurement accuracy associated with experimental factors and correlation algorithms is discussed and some useful recommendations for reducing measurement errors are also offered. Then, the utilization of DIC in different manufacturing fields (e.g. cutting, welding, forming and additive manufacturing) is summarized. Finally, the current challenges and prospects of DIC in intelligent manufacturing are discussed.
\end{abstract}

Keywords Digital image correlation, Kinematic fields, Manufacturing process, Sensing

Paper type Book review

\section{Introduction}

For decades, more and more attention is being paid to intelligent manufacturing with the capability of manufacturing various products with minimal supervision and assistance from operators (Zhou et al., 2018). Vigorously developing intelligent manufacturing is of great significance for improving production efficiency, intelligentization and green growth of manufacturing. However, the realization of intelligent manufacturing still faces many challenging issues. As one of the core issues in intelligent manufacturing, measurement technology is an essential part that provides intelligent perception, which is the basis of detection, control and decision in the whole manufacturing process. So far, in-depth works have been done for developing measurement technology in the manufacturing field by many researchers, and a variety of innovative methods have been explored, mainly focusing on the measurement of kinematic fields to obtain displacement and strain information, such as mechanical tests (fatigue, fracture, impact, etc.) and materials processing (welding, forming, cutting, etc.). Several commonly employed methods for the displacement or deformation measurement could be categorized as (1) the finite difference of the rigid-body displacement at the edge of the observed structure (Harangus and Kakucs, 2014) and (2) glued strain gages (Little, 1992; Zhang, 2019).

(C) Pingan Zhu, Chao Zhang and Jun Zou. Published in Journal of Intelligent Manufacturing and Special Equipment, Published by Emerald Publishing Limited. This article is published under the Creative Commons Attribution (CC BY 4.0) licence. Anyone may reproduce, distribute, translate and create derivative works of this article (for both commercial and non-commercial purposes), subject to full attribution to the original publication and authors. The full terms of this licence may be seen at http:// creativecommons.org/licences/by/4.0/legalcode

The authors convey their deepest gratitude to the peer reviewers for their careful work and thoughtful suggestions that have helped improve the paper substantially.

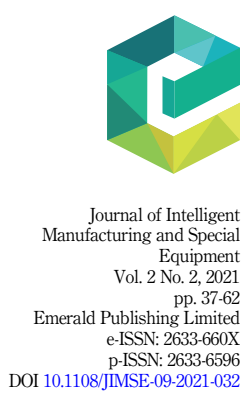


JIMSE

2,2

38

In the first scenario, the desired displacement measurements are obtained by the relative motion of the two arms of the extensometer, either on the tester itself or glued or clamped to a specific point on the surface. Except from the practical issues of realization, the information acquired should be averaged between the points where the motion is known. Since the local phenomena are negligible, the position and size of the detection would be unknown. In the second case, it is clear that the utilization of strain gages is a contact measurement with the following several inherent limitations. (1) For small specimens or soft materials, the additional stiffness and weight of the strain gauges can seriously affect the measurement results, since the gages are glued to the surface of the specimen during the measurement. (2) Strain gages as a point measurement method cannot obtain full-field information and are not applicable to the measurement of non-uniform deformation. (3) The limited measurement range of strain gages means that the resistive wire of strain gages will fail when measuring large deformations (Zhang, 2019). In contrast, optical measurements, as a non-contact full-field measurement method, can handle the above mentioned issues. Many optical measurement methods allow quantization of displacement, including (1) interferometric techniques such as holographic interferometry (Georges, 2019), speckle scattering interferometry (Asseban et al., 2000) and moire interferometry (Walker, 1994) and (2) non-interferometric techniques such as DIC and particle image velocimetry (PIV) (Grant, 1997).

Usually, deformation is obtained by interpreting the interference in interferometric optical measurements. More concretely, interferometry is achieved by projecting a specific pattern (grid, line or speckle) onto the exact same pattern that is painted on the object to be imaged. The superposition discrepancy represents mainly local displacements and produces an image where the deconstructed displacements can be quantified. In addition, laser interferometry is performed by recording the phase change of scattered light waves from the surface of the object under test. Given the high-speed scanning, laser interferometry can be applied to point measurements or in situ measurements. The technique usually requires a coherent light source, i.e. a laser. However, it is critical to meet environmental requirements when performing interferometric measurements, which are usually performed in vibration-isolated optical platforms. Therefore, these techniques are commonly used in laboratories and are difficult to apply in industrial applications due to the complexity of the environment. For non-interferometric optical measurements, only a noncoherent light source is necessary to acquire the deformation of the surface by comparing the grayscale intensity changes; thus, they are more accessible than interferometric optical measurement methods (Zhang, 2019). The DIC method is a representative noninterferometric measurement method that has been extensively used to measure the deformation of solid materials. A typical workflow of DIC displacement measurement is shown in Figure 1 (Hild and Roux, 2006; Pan et al., 2009, 2018; Schreier et al., 2009; Dong and Pan, 2017; Pan, 2018). In general, for the manufacturing field, the DIC technique is a promising optical method that can overcome most of the above limitations. In addition, the PIV technique is commonly used for flow-field analysis.

This work is intended to provide a comprehensive review of the DIC technique for those who are interested in performing the DIC technique in the area of manufacturing. A historical overview of DIC is introduced in Section 2. Section 3 elaborates on the main advantages of DIC. The measurement setup and basic principles of DIC are outlined in Section 4 and 5, respectively. The influencing factors on the measurement accuracy of DIC are discussed in Section 6 and some recommendations for reducing measurement errors are correspondingly offered. Section 7 focuses on the applications of DIC in different manufacturing fields. At the end of this review, the persistent challenges and future direction of DIC for intelligent manufacturing are discussed. 


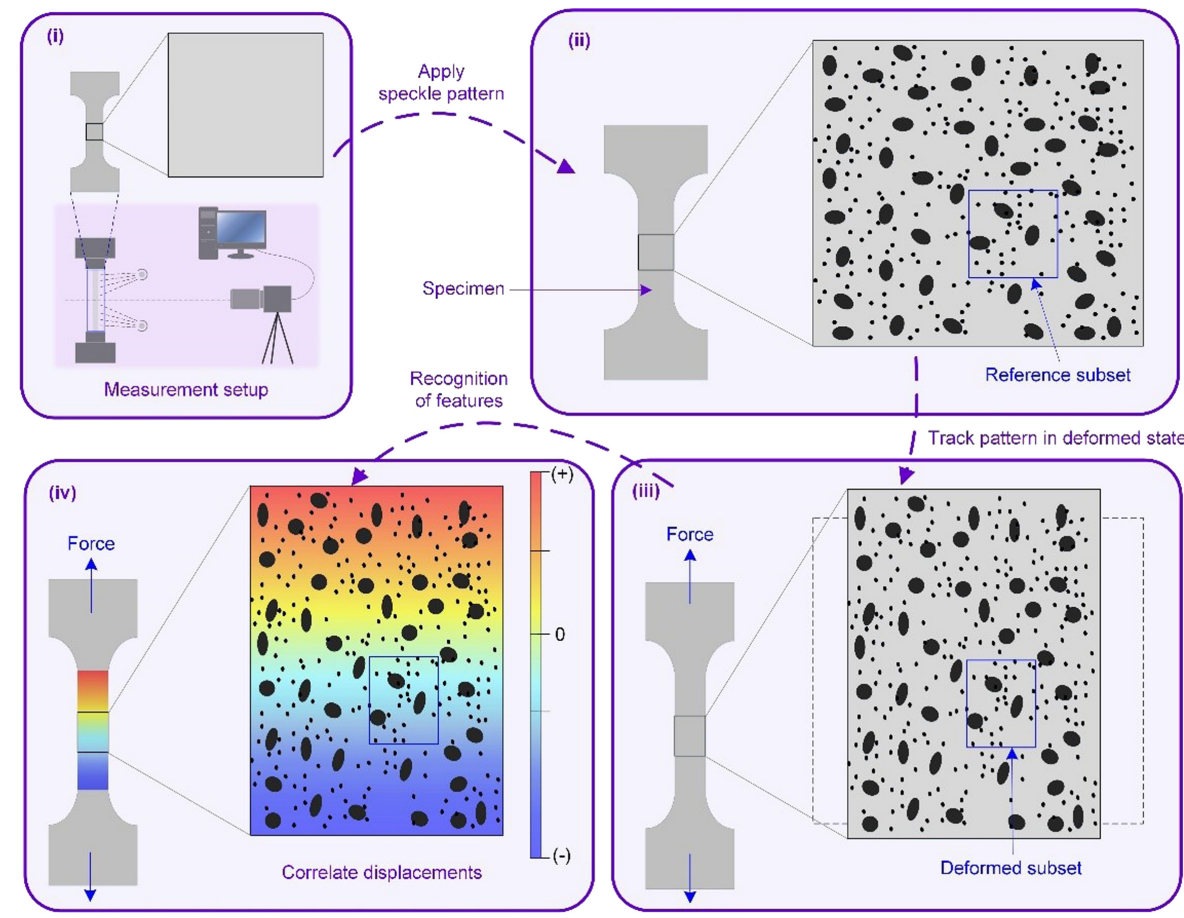

\section{Historical overview of DIC}

In the early 1980s, the idea of the DIC technique was first proposed by Yamaguchi (1981a, b) and Bruck et al. (1989). Yamaguchi studied the deformation of small objects by calculating the correlation function of the intensity of speckle illumination. A few years later, Peters and Ranson adopted iterative operations for digital images of objects acquired from before and after deformation. They derived the extreme values of the correlation coefficients by calculating the correlation coefficients and their derivatives with displacement to obtain the corresponding displacements. Many researchers have done intensive work on how to fasten the speed and improve the accuracy of the calculation. Peters et al. applied the DIC technique for the first time to measure the displacement of a stiff body (Peters et al., 1983). Sutton et al. improved the correlation search methodology and used a combination of coarse and fine correlation to enhance the computational speed (Sutton et al., 1983). HE et al. investigated the accuracy of the DIC technique and improved the theory of this measurement method (He et al., 1984). Sutton et al. analyzed the measurement error arising from the sub-pixel recovery process and proposed reasonable methods for dealing with sub-pixel recovery (Sutton et al., 1986). Bruck et al. adopted the Newton-Raphson (NR) iterative algorithm based on binary cubic-spline interpolation sub-pixel reconstruction to solve the DIC problem, which significantly improved the DIC method (Bruck et al., 1989). Vendroux and Knauss used the first-order derivative approximation for the Hessian matrix, replacing the NR algorithm with the Gauss-Newton algorithm, which improved the computational efficiency by eliminating the process of calculating the second-order gray gradient while ensuring the computational accuracy (Vend and Knauss, 1998; Vendroux and Knauss, 1998; Vendroux et al., 1998).

Since 2000, DIC technology has entered a boom period with the continuous development of computer technology and the increasing accessibility of imaging devices (Pan, 2018).
Book review

Figure 1.

The typical workflow of DIC displacement measurement 
JIMSE

2,2

40

Research in this phase has focused on (1) new imaging systems (Schreier et al., 2004; Berfield et al., 2006; Louis et al., 2007) and the characterization and correction of device-induced measurement errors (Jin and Bruck, 2005; Luo, 2006); (2) speckle pattern fabrication and quality evaluation (Reu, 2014; Lecompte et al., 2006; Stoilov et al., 2012; Zhang et al., 2018); (3) development of correlation algorithm (Tong, 2005; Pan, 2009) and (4) evaluation of DIC measurement errors (Lecompte et al., 2006; Haddadi and Belhabib, 2008; Sutton et al., 2008; Bornert et al., 2009; Wang et al., 2009). For instance, Peng et al. used a computer to generate digital images with random speckles according to Gaussian intensity distribution in order to simulate the displacement speckle pattern of the object before and after deformation in 2001 (Zhou, 2001). In this work, an iterative, spatial-gradient-based algorithm was developed that requires the calculation of only first-order spatial derivatives of the images and thus reduces the computation complexity. In 2006, Pan et al. conducted a detailed and systematic study and evaluation of the systematic errors and random errors of the existing sub-pixel displacement measurement algorithms in which a conclusion that the NR algorithm based on spatial iterative cross-correlation $(\mathrm{CC})$ is more accurate but much slower than other algorithms (Pan et al., 2006). Later, Pan et al. made a detailed analysis of the selection of subset size and quantitative evaluation of speckle quality, and also mathematically derived the theoretical model of displacement measurement accuracy of the two-dimensional DIC method (Pan et al., 2008). More recently, Shao et al. proposed a pixel-selection strategy to improve the computational efficiency of DIC further, which allows a real-time deformation measurement with a large subset (Shao et al., 2020).

Many essential advances have been made, making DIC one of the most popular and practical measurement techniques. Meanwhile, over the years, the DIC technique has been extended from the initial displacement analysis in metal detection to many other fields such as biomechanics (Palanca et al., 2016), civil engineering (Ramos et al., 2015), aerospace (Chu and Poudel, 2014), electronics (Niu et al., 2018) and so on. In addition, with the advent of atomic force microscopy, transmission electron microscopy and scanning tunneling microscopy, DIC is widely used for microscopic observation (Budyn et al., 2012). Specifically, these applications are concentrated in the areas (Zhang, 2019) as follows:

(1) Measure deformation properties and mechanical properties of diverse materials (e.g. metals, composites, polymers and wood) under mechanical and thermal loads. Zink et al. first used the DIC technique to study the mechanical properties of wood (Audrey and Robert, 1983). Choi et al. applied the DIC technique to measure the deformation of concrete under compressive loading and to obtain the failure performance of concrete (Choi and Shah, 1997). Bastawros et al. applied the DIC technique to obtain three stages of the deformation process of the aluminum alloy foam under compressive loading (Bastawros et al., 2000). Chevalier et al. studied the axial tensile tests of a kind of polymer by DIC (Chevalier et al., 2001).

(2) From displacement and strain information acquired by the DIC technique, various material parameters such as modulus of elasticity, Poisson's ratio, coefficient of thermal expansion, stress intensity factor, fracture toughness, etc. can be evaluated. For instance, Zhang et al. studied the variation of Poisson's ratio of the arterial vessels with axial strain by DIC (Zhang et al., 2002). Yoneyama (2006) and Réthoré et al. (2005) adopted the DIC technique to measure the stress intensity factor at the crack tip.

(3) The full-field deformation data of the object surface obtained by the DIC technique are utilized for theoretical analysis and numerical simulations (Knauss et al., 2003; Cho et al., 2005; Sabaté et al., 2006). Thus, theory, simulation and experiment can be well linked to achieve in-depth and thorough analysis. 


\section{Advantages of DIC technique}

The emergence and development of DIC were made possible by the rapid development of camera technology and the computing power of personal computers. The DIC method has a number of attractive advantages over other traditional measurement techniques as follows (Zhang, 2019):

Simple experimental setup: The main device has only one camera, such as a charge-coupled device (CCD) or a complementary metal-oxide-semiconductor (CMOS), which is intended to record images of the surface of an object during its movement.

Simple specimen preparation: If the surface of the specimen has a natural texture with random gray intensity distribution, the specimen can be used directly, where the natural texture can be considered as speckles for easy tracking in the DIC. If not, the sample surface can be easily prepared by spraying black and/or white paint as speckles.

Strong environmental adaptability: The DIC technique does not require a laser source; typically, a white light source or natural light is sufficient for the measurement. Preferably, the illumination of the imaging scene remains constant throughout the recording process, while relatively small variations in illumination can also be tolerated as long as the surface texture is unique. In addition, DIC can be applied to complex operating conditions such as high temperature, high pressure, high-speed impact, vibration, etc. Therefore, DIC technology is well suited for industrial applications such as manufacturing processes.

Wide measurement range: The DIC method is expected to directly process digital images that can be acquired with various high-resolution digital acquisition devices, such as optical microscopes (Sun et al., 1997; Pitter et al., 2002), laser scanning confocal microscope (Berfield et al., 2006; Franck et al., 2007), scanning electron microscopy (Kang et al., 2005; Wang et al., 2006), transmission electron microscopy (Calvié et al., 2014), atomic force microscopy (Chang et al., 2005; Sun and Pang, 2006), scanning tunneling microscope (Vend and Knauss, 1998; Vendroux and Knauss, 1998; Vendroux et al., 1998) and satellites (Leprince et al., 2007). All these types of images can be analyzed by the DIC method, which makes it possible to measure displacements from microscopic to macroscopic scales using the DIC method.

\section{DIC measurement setup}

\subsection{Specimen preparation}

Surface patterns on the test specimen are essential for DIC, i.e. there must be sufficiently recognizable patterns on the surface of the measured object. As a carrier of displacement information, the surface pattern should possess the features of high contrast, randomness, isotropy and stability in order to achieve accurate DIC measurements (Dong and Pan, 2017). Except for a few specimens with natural texture on their surfaces, such as the grains on the metal surface or milling streaks, artificial speckles made by spraying black and/or white paints are required. Spray painting is currently the most common way to obtain speckle pattern, but manual spray is hard to control the quality of speckle. Some examples of speckles are schematically shown in Figure 2. Isotropic patterns ensure that displacements can be identified in each direction of the plane. The high-contrast speckle pattern makes sure that there is maximum gradient. An illustration of the best pattern is shown in Figure 2(d). The other three patterns are not good for performing DIC. In addition to spray painting, printing a speckle pattern is another way to firmly attach the printed speckle sticker to the specimen surface and deform it with the specimen. Nevertheless, if the printed speckle slips against the specimen surface due to creases and bubbles, the experimental data obtained from the image no longer reflect the actual deformation of the specimen (Zhang, 2019).

\subsection{Image acquisition system}

For a typical DIC image acquisition system, as shown in Figure 3, there are four main elements as follows: (1) a surface speckled specimen; (2) a silicon-based camera (CCD or 


\section{JIMSE \\ 2,2}

42

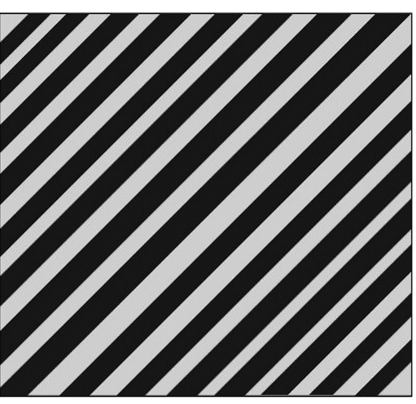

Repetitive, anisotropic, high-contrast

(a)

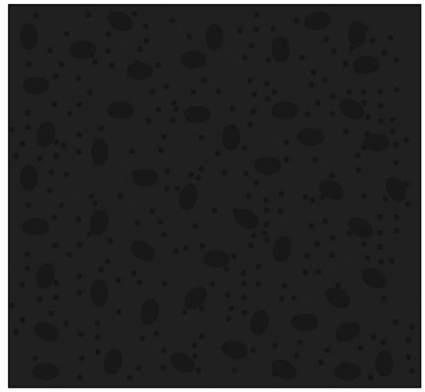

Non-repetitive, isotropic, low-contrast

(c)

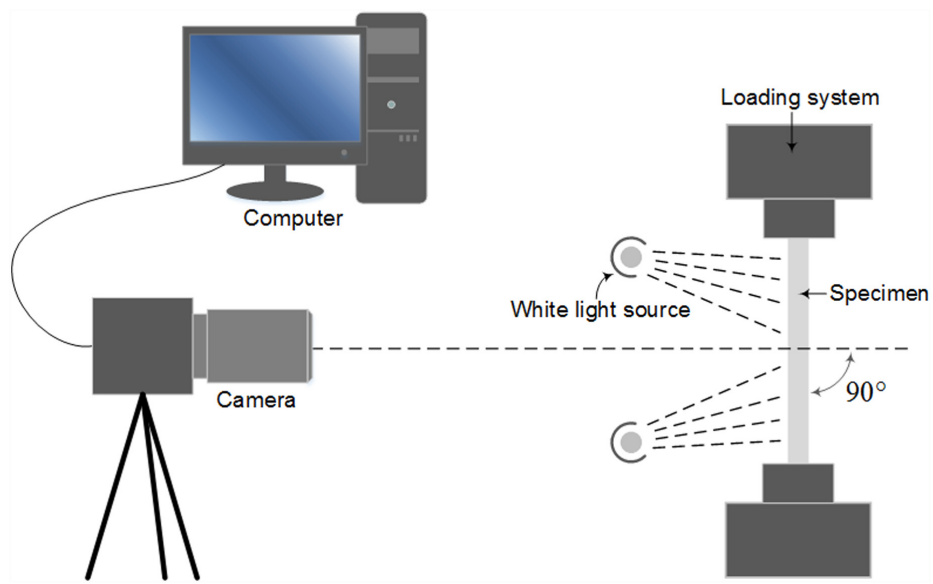

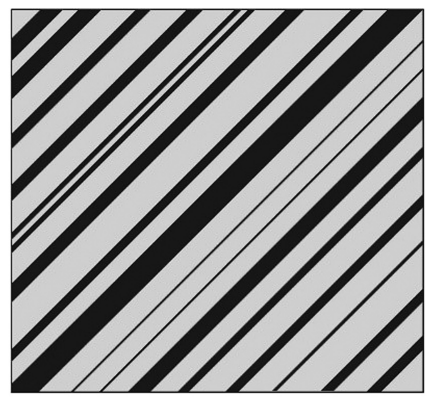

Non-repetitive, anisotropic, high-contrast

(b)

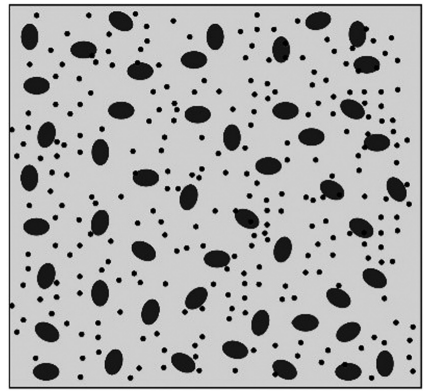

Non-repetitive, isotropic, high-contrast

(d)
Figure 3.

Typical optical image acquisition system

CMOS); (3) a computer for data acquisition and (4) a white light source. More specifically, the camera is placed on a tripod so that its optical axis is perpendicular to the sample surface. The white light source is intended to provide a good view of the surface of the specimen with a high-contrast grayscale intensity distribution. When the specimen is loaded, the camera records images of the specimen during the deformation process. The acquired images are 
then processed $a$ posteriori by a computer to obtain the displacement field. In addition, the strain field can be inferred from the displacement field (Zhang, 2019).

\section{Basic principles of DIC}

The fundamental principle of DIC method is to choose a reference subset with $(2 k+1) \times(2 k+1)$ pixels $(k$, the number of pixels) in the reference image $f(x, y)$, i.e. the image acquired before deformation, and then to determine the corresponding subset location in the deformed image $g\left(x^{\prime}, y^{\prime}\right)$ based on the gray level information of the subset. It can be noted that this justifies why the surface pattern cannot be repeated, otherwise the determined solution may not be unique. Therefore, the displacement and strain can be obtained based on the changes in the position and shape of a subset of the two images (Zhang, 2019).

To achieve the above goal, a correlation calculation is required in DIC. First, a suitable set of variables should be found to represent the displacement and deformation of subsets in the reference image and the deformation image. Second, a mathematical criterion should be established to evaluate the degree of correspondence between the two subsets: one in the reference image and the other in the final strain and displacement image. Finally, the displacements and strains of the subsets are obtained by iterative calculations using an efficient search algorithm.

\subsection{Shape functions}

As shown in Figure 4, a point $P\left(x_{0}, y_{0}\right)$ is in the reference image. To calculate the displacement of point $P$, a reference subset centered on point $P\left(x_{0}, y_{0}\right)$ of the reference image is utilized to track its corresponding position in the deformed image. The reason for choosing a square subset instead of individual pixels for matching is that the subset containing a wider range of grayscale variations will be distinguished from other subsets and can, therefore, be identified more uniquely in the distorted image (Zhang, 2019).

The subset centered at point $P\left(x_{0}, y_{0}\right)$ moves to a location near point $P^{\prime}\left(x_{0}{ }^{\prime}, y_{0}{ }^{\prime}\right)$. The displacement of point $P$ on the $x$ and $y$ axes is $u$ and $v$, respectively. Thus,

$$
\left\{\begin{array}{l}
x_{0}^{\prime}=x_{0}+u \\
y_{0}^{\prime}=y_{0}+v
\end{array}\right.
$$

Meanwhile, the point $Q\left(x_{i}, y_{i}\right)$ represents any point around the point $P$ in the reference subset; the positional relationship between point $P$ and point $Q$ is given as follows:
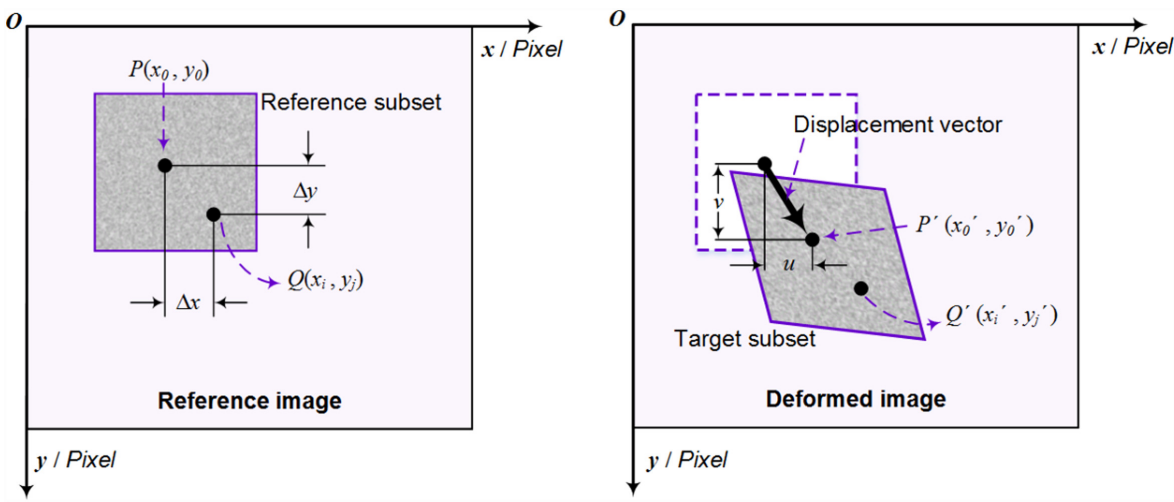

Figure 4. Schematically illustration of a reference square subset before deformation and a targeted subset after deformation 
JIMSE

2,2

$$
\left\{\begin{array}{l}
x_{i}=x_{0}+\Delta x \\
y_{i}=y_{0}+\Delta y
\end{array}\right.
$$

where $\Delta x$ and $\Delta y$ is the distance between point $P$ and point $Q$ on the $x$ and $y$ axes, respectively. If the point $Q$ in the reference subset moves to point $Q^{\prime}\left(x_{i}^{\prime}, y_{i}^{\prime}\right)$, an equation can be given as follows:

$$
\left\{\begin{array}{l}
x_{i}^{\prime}=x_{i}+u_{Q} \\
y_{i}^{\prime}=y_{i}+v_{Q}
\end{array}\right.
$$

Generally, the material imaged in the reference subset allows translation, rotation and shear. Thus, the displacement of point $Q$ on the $x$ and $y$ axes $\left(u_{Q}\right.$ and $\left.v_{Q}\right)$ can be given by the firstorder displacement gradient of point $P$ :

$$
\left\{\begin{array}{l}
u_{Q}=u+\frac{\partial u}{\partial x} \Delta x+\frac{\partial u}{\partial y} \Delta y \\
v_{Q}=v+\frac{\partial v}{\partial x} \Delta x+\frac{\partial v}{\partial y} \Delta y
\end{array}\right.
$$

Substituting Eq. (4) into Eq. (3) yield:

$$
\left\{\begin{array}{l}
x_{i}^{\prime}=x_{i}+u+\frac{\partial u}{\partial x} \Delta x+\frac{\partial u}{\partial y} \Delta y \\
y_{i}^{\prime}=y_{i}+v+\frac{\partial v}{\partial x} \Delta x+\frac{\partial v}{\partial y} \Delta y
\end{array}\right.
$$

Since point $Q$ is any point in the subset, it is found from Eq. (5) that displacement of the subset can be expressed by the displacement of the center point in the subset and its four derivatives $\left(u, v, \frac{\partial u}{\partial x} \frac{\partial u}{\partial y}, \frac{\partial v}{\partial x}, \frac{\partial v}{\partial y}\right)$.

\subsection{Correlation criterion}

In order to assess the degree of similarity between the reference and deformation subsets, a correlation criterion should be given. Different correlation criteria have been proposed so far. Usually, the correlation criteria consist of two groups: CC criteria and the sum of squared differences (SSD) criteria. Three commonly used CC criteria are as follows (Zhang, 2019):

(1) Cross-correction (CC):

$$
C_{\mathrm{CC}}=\sum_{-k}^{k} \sum_{-k}^{k}\left[f(x, y) \cdot g\left(x^{\prime}, y^{\prime}\right)\right]
$$

(2) Normalized cross-correction (NCC):

$$
C_{\mathrm{NCC}}=\frac{\sum_{-k}^{k} \sum_{-k}^{k}\left[f(x, y) \cdot g\left(x^{\prime}, y^{\prime}\right)\right]}{\left[\sum_{-k}^{k} \sum_{-k}^{k} f^{2}(x, y)\right]^{1 / 2} \cdot\left[\sum_{-k}^{k} \sum_{-k}^{k} g^{2}\left(x^{\prime}, y^{\prime}\right)\right]^{1 / 2}}
$$


(3) Zero-normalized cross-correction (NCC):

$$
C_{\mathrm{ZNCC}}=\sum_{-k}^{k} \sum_{-k}^{k}\left\{\frac{\left[f(x, y)-f_{m}\right] \cdot\left[g\left(x^{\prime}, y^{\prime}\right)-g_{m}\right]}{\left\{\sum_{-k}^{k} \sum_{-k}^{k}\left[f(x, y)-f_{m}\right]^{2}\right\}^{1 / 2} \cdot\left\{\sum_{-k}^{k} \sum_{-k}^{k}\left[g\left(x^{\prime}, y^{\prime}\right)-g_{m}\right]^{2}\right\}^{y_{2}}}\right\}
$$

where $f_{m}=\frac{1}{(2 k+1)^{2}} \sum_{-k}^{k} \sum_{-k}^{k} f(x, y)$ and $g_{m}=\frac{1}{(2 k+1)^{2}} \sum_{-k}^{k} \sum_{-k}^{k} g\left(x^{\prime}, y^{\prime}\right)$.

Three commonly used SSD criteria are given as follows:

(1) Sum of squared differences (SSD):

$$
C_{\mathrm{SSD}}=\sum_{-k}^{k} \sum_{-k}^{k}\left[f(x, y)-g\left(x^{\prime}, y^{\prime}\right)\right]^{2}
$$

(2) Normalized sum of squared differences (NSSD):

$$
C_{\mathrm{NSSD}}=\sum_{-k}^{k} \sum_{-k}^{k}\left[\frac{f(x, y)}{\left\{\sum_{-k}^{k} \sum_{-k}^{k}[f(x, y)]^{2}\right\}^{1 / 2}}-\frac{g\left(x^{\prime}, y^{\prime}\right)}{\left\{\sum_{-k}^{k} \sum_{-k}^{k}\left[g\left(x^{\prime}, y^{\prime}\right)\right]^{2}\right\}^{1 / 2}}\right]^{2}
$$

(3) Zero-normalized sum of squared differences (ZNSSD):

$$
C_{\mathrm{ZNSSD}}=\sum_{-k}^{k} \sum_{-k}^{k}\left\{\frac{f(x, y)-f_{m}}{\left\{\sum_{-k}^{k} \sum_{-k}^{k}\left[f(x, y)-f_{m}\right]^{2}\right\}^{1 / 2}}-\frac{g\left(x^{\prime}, y^{\prime}\right)-g_{m}}{\left\{\sum_{-k}^{k} \sum_{-k}^{k}\left[g\left(x^{\prime}, y^{\prime}\right)-g_{m}\right]^{2}\right\}^{1 / 2}}\right\}
$$

The basic principles of these correlation criteria are the same. Here, the normalized CC (NCC) is used to be analyzed as an example. The definition of NCC is given as follows:

$$
C\left(x, y, u, v, \frac{\partial u}{\partial x}, \frac{\partial u}{\partial y}, \frac{\partial v}{\partial x}, \frac{\partial v}{\partial y}\right)=\frac{\sum_{-k}^{k} \sum_{-k}^{k}\left[f(x, y) \cdot g\left(x^{\prime}, y^{\prime}\right)\right]}{\left[\sum_{-k}^{k} \sum_{-k}^{k} f^{2}(x, y)\right]^{1 / 2} \cdot\left[\sum_{-k}^{k} \sum_{-k}^{k} g^{2}\left(x^{\prime}, y^{\prime}\right)\right]^{1 / 2}}
$$

where $x, y$ are the coordinates of the center point in the subset and $x^{\prime}, y^{\prime}$ are the coordinates of any point in the subset and can be obtained by Eq. (5). When the maximum value is achieved (i.e. $C=1$ ), the subset in the deformed image is correlated with the reference subset in the reference image.

In correlation calculation, an optimization algorithm is normally adopted to find the minimum value. Thus, a correlation factor $\mathrm{S}$ is introduced as follows: 
JIMSE

2,2

46

$$
S=1-C=1-\frac{\sum_{-k}^{k} \sum_{-k}^{k}\left[f(x, y) \cdot g\left(x^{\prime}, y^{\prime}\right)\right]}{\left[\sum_{-k}^{k} \sum_{-k}^{k} f^{2}(x, y)\right]^{1 / 2} \cdot\left[\sum_{-k}^{k} \sum_{-k}^{k} g^{2}\left(x^{\prime}, y^{\prime}\right)\right]^{1 / 2}}
$$

It is clear from Eq. (13) that the smaller the $S$ value, the greater the correlation between the two subsets. Also, it is worth to note that the correlation values calculated by the ZNCC or ZNSSD correlation criterion are changeless if only an offset and a scale in lighting, i.e. linear transformation of the target subset gray intensity. Thus, the ZNCC or ZNSSD criterion could accommodate changes in lighting. Meanwhile, the NCC or NSSD correlation criterion is insensitive to the linear scale in lighting but sensitive to offset of the lighting. The CC or SSD correlation criterion is sensitive to all changes in lighting, as shown in Table 1.

\subsection{Search algorithm}

A vector $J$ is introduced to represent the displacement of the subset, which is given as follows:

$$
J=\left\{x, y, u, v, \frac{\partial u}{\partial x}, \frac{\partial u}{\partial y}, \frac{\partial v}{\partial x}, \frac{\partial v}{\partial y}\right\}
$$

$J_{i}(i=1,2,3,4,5,6)$ is utilized to represent the $u, v, \frac{\partial u}{\partial x}, \frac{\partial u}{\partial y}, \frac{\partial v}{\partial x}, \frac{\partial v}{\partial y}$. Therefore, the correlation calculation is used to find the minimum of the objective function $S\left(J_{1}, J_{2}, J_{3}, J_{4}, J_{5}, J_{6}\right)$. Obviously, the necessary condition for $S$ to reach its minimum value is that its first-order derivative is zero.

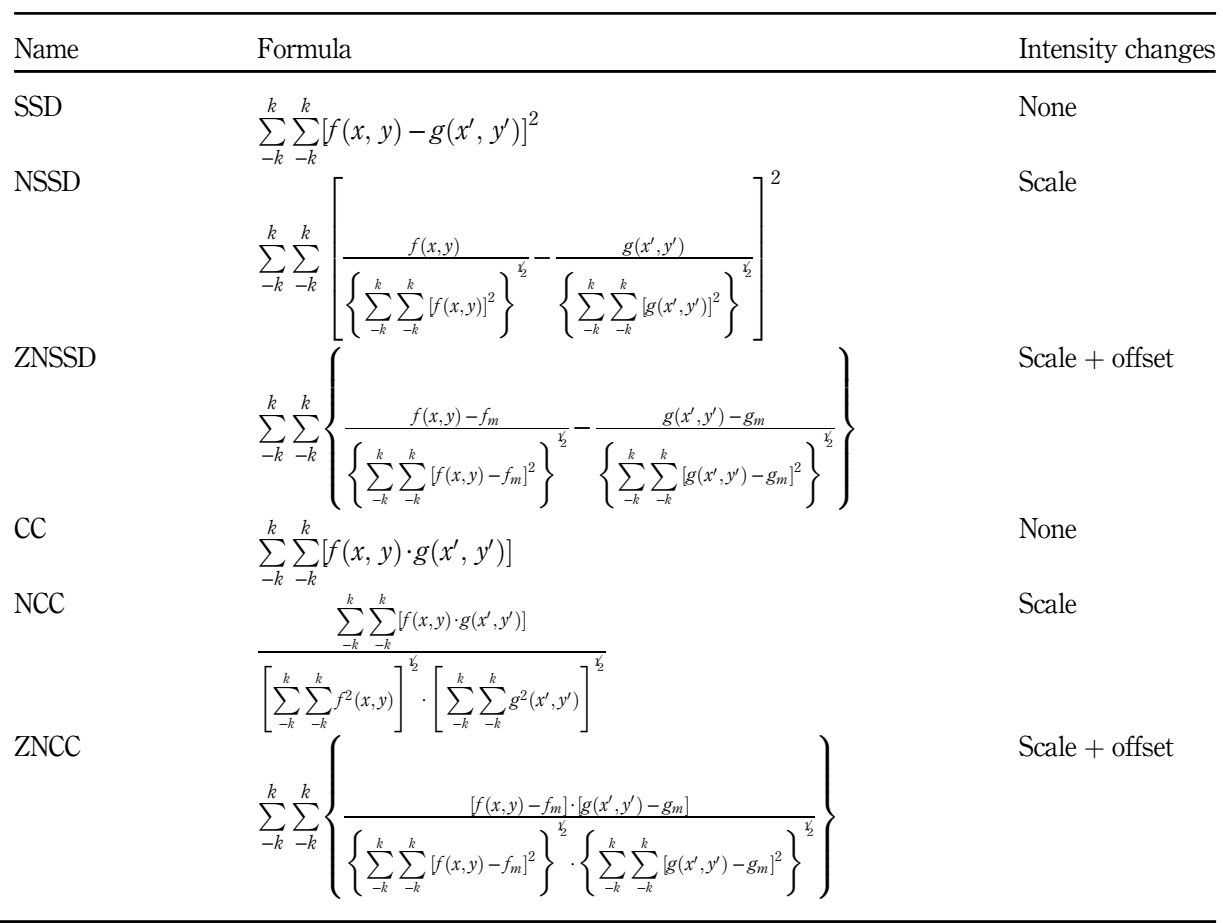

Table 1.

Summary of the common optimization criterion 


$$
\frac{\partial S}{\partial J_{i}}=0
$$

For this optimization issue, there are many numerical methods such as NR, etc. When the $S$ reaches a minimum, we can obtain the displacement and deformation of the subset from the $J_{i}$ values (Zhang, 2019).

The process described above is the basic principle of the local image correlation method in which a small region of interest, i.e. the aforementioned reference subset, is selected for processing as shown in Figure 4. The main drawback of the approach is that the process could cause large fluctuations (from speckle and the variation of lighting) from one image to the other.

The main limitation of the local method is that the process can induce large fluctuations from one image to the other (from speckle and the variation of lighting). The global image correlation method is superior to the local method and avoids the limitations of the local method described above (Hild and Roux, 2012). The global method relies on the entire image to determine the displacement field based on mesh and shape functions (Besnard et al., 2006).

\section{Errors affecting DIC measurements}

Obviously, the estimation of the errors caused by different error sources is important to improve the measurement accuracy of DIC. DIC techniques rely on a complex measurement chain, so the measurement errors could stem from each of its elements. Generally, the cause of the measurement errors could be categorized into two parts: one is related to the correlation principle itself and the other is associated with the measurement devices and the working environment. In this section, different sources of measurement error are analyzed in detail and recommendations for avoiding these measurement errors are also offered.

Specifically, the errors related to the correlation principle itself could stem from (1) the quality of the speckle pattern, (2) the step size and the subset size and (3) the correlation algorithm. First, the speckle pattern on the specimen surface is the carrier of the deformation information of the specimen. The DIC collects the deformation information of the specimen by identifying and tracking the speckle field on the surface of the specimen. Thus, the quality of the speckle pattern is closely related to the measurement accuracy of DIC methods. The traditional evaluation parameters of speckle quality focus on reducing random errors, so fine speckle is normally used in experiments. However, speckle that is too fine will increase the interpolation deviation, which results in unsatisfactory experimental results. Researchers have tried to theoretically deduce the error related to the quality of the speckle pattern in DIC and the various evaluation mechanisms of the quality of the speckle pattern are given by them, such as mean speckle size (Lecompte et al., 2006), subset entropy (Yaofeng and Pang, 2007) and the sum of the square of subset intensity gradients (Pan et al., 2008) etc. As mentioned before, the creation of a surface pattern with the features of high contrast, randomness, isotropy and stability is essential to improve measurement accuracy.

Additionally, as a user-defined input parameter, the chosen step and subset size are also critical to the measurement accuracy. In order to achieve a reliable analysis of correlation, the subset size should be big enough so that there is a sufficiently distinctive intensity pattern included in the subset to distinguish itself from other subsets. Meanwhile, larger subsets require more computation time. On the other hand, whereas, the underlying deformation field of a small subset could be accurately approximated by the first- or second-order subset shape function, but a large subset size tends to lead to large errors in the approximation of the underlying deformations. Therefore, the chosen subset size should be small so as to 
JIMSE

2,2

guarantee reliable results of displacement measurement. Based on the aforementioned two conflicting requirements, there should be a trade-off in the choice of subset size (Pan et al., 2009). Besides, the step size has a stronger effect on spatial resolution than the subset size, i.e. smaller step sizes yield more DIC data points and thus higher spatial resolution.

In addition, the correlation algorithm affects the measurement accuracy of DIC methods, influencing factors including shape function, interpolation scheme, correlation criterion, etc. When the subset size is small, the first-order shape function or the second-order shape function could accurately describe the displacement of the points in the subset. However, they are obtained by expending the displacement function of points in the subset at the center of the subset using the first-order or second-order Taylor series expansion, which are an approximation of actual values. Scherier et al. analyzed the systematic errors introduced by the shape function in the DIC method (Schreier and Sutton, 2002). They concluded that although the second-order shape function cannot improve the calculation accuracy of the displacement derivative, it could reduce the systematic error introduced by the shape function. And in some cases, the second-order shape function could describe the deformation more accurately than the first-order shape function. Besides, measurement error could also be introduced by the use of interpolation schemes. In the DIC, so far, a variety of interpolation algorithms have been utilized to perform the grayscale reconstruction of sub-pixel, including bilinear interpolation (Bruck et al., 1989), bicubic interpolation (Jin, 2005) etc. However, subpixel interpolation could only approximately restore the grayscale of the image and cannot acquire the completely correct value of grayscale. Schreier et al. investigated the impact of interpolation phase error on the matching error in DIC and high-order interpolation methods with smaller phase errors are recommended to reduce these systematic errors (Schreier, 2000). Additionally, the correlation criterion also has an effect on the measurement accuracy due to the variation in grayscale in the actual environment, such as changes in lighting. As mentioned before, the ZNCC or ZNSSD criterion could accommodate changes in lighting and thus are recommended in the correlation criterion.

On the other hand, the measurement errors caused by the measurement devices and the working environment cannot be despised either. First of all, the DIC method obtains the surface image of the specimen through the optical lens, whereas the common optical lens inevitably has image distortion. Therefore, it is helpful to estimate the distortion of the camera lens for improving the measurement accuracy. The correction of lens distortion could be divided into two categories: one is that correcting the distortion by solving the distortion coefficient of the known distortion model (Yoneyama, 2006) and the other type is based on camera calibration (Helm, 1996), which is characterized by other parameters of the camera being solved together. Notably, the former method is mostly used for two-dimensional DIC, while the latter method is suitable for distortion correction and camera parameter calibration of three-dimensional DIC methods. Additionally, the disturbances from the external environment, such as light, vibration and airflow, also affect the measurement accuracy of DIC methods. In order to reduce the measurement error caused by the environment, some interferences such as monochromatic light are employed in the experiment and some algorithmic methods are also proposed by researchers. For instance, Pan et al. proposed a method that pre-smoothing the speckle images with a $5 \times 5$ pixels Gaussian low-pass filter before correlation analysis in which both numerical simulations and experiments reveal that the proposed technique could reduce the bias error for both noisy and noiseless images. The significant reduction of the bias error by using Gaussian pre-filtering is shown in Figure 5 (Pan, 2013). Except for disturbances from the environment, the undesired noise could also stem from measurement devices, such as photon noise and thermal noise. For these noises introduced by measurement devices, the use of a high-quality camera with better imaging effects could be helpful and also larger subset could be selected to reduce the influence of such noise. 


\section{DIC applications in manufacturing}

Cutting: DIC technique has a broad application prospect in the research of cutting mechanism, notable examples including (1) observing the formation process of continuous chip and the propagation of crack via DIC, which provides an intuitive understanding of the cutting process; (2) DIC technique could clearly obtain physical quantities such as shear angle, tool radius, tool contact length, etc., which could be used as a reliable basis of theoretical research; (3) further processing from the displacement field in the cutting deformation area could obtain a series of useful variables and results such as the strain field, strain rate field, plastic strain field, etc. and (4) DIC technique offers an experimental method for deepening the understanding of the cutting behavior of new composite materials whose constitutive parameters are difficult to obtain, etc.

To date, the research of the cutting process by using the DIC technique is mainly focused on two conditions: studying low-speed cutting with a high-speed camera and high-speed cutting observation with a double-frame camera. For instance, Davis et al. investigated the chip-formation mechanism using the DIC technique with an in situ high-speed camera in which the chip morphology is characterized according to its cross-sectional geometry using different cutting speeds (Davis et al., 2018a). Also, Davis et al. further studied the strain and strain rate progression with the primary shear zone during chip formation based on the accurate measurement provided by the DIC technique with a high-speed camera. The experimental setup is shown in Figure 6(a) and (b), and shear strain distribution along the chip flow direction at a cutting speed of $0.5 \mathrm{~m} / \mathrm{s}$ is shown in Figure 6(d) (Davis et al., 2018b). Due to the limitations of the performance of high-speed cameras, only the cutting process with relatively low-cutting speeds could be analyzed by using DIC, which hinders the application of DIC in practice. Therefore, some researchers have adopted a double-frame camera to observe the cutting process. The exposure time of the double-frame camera could
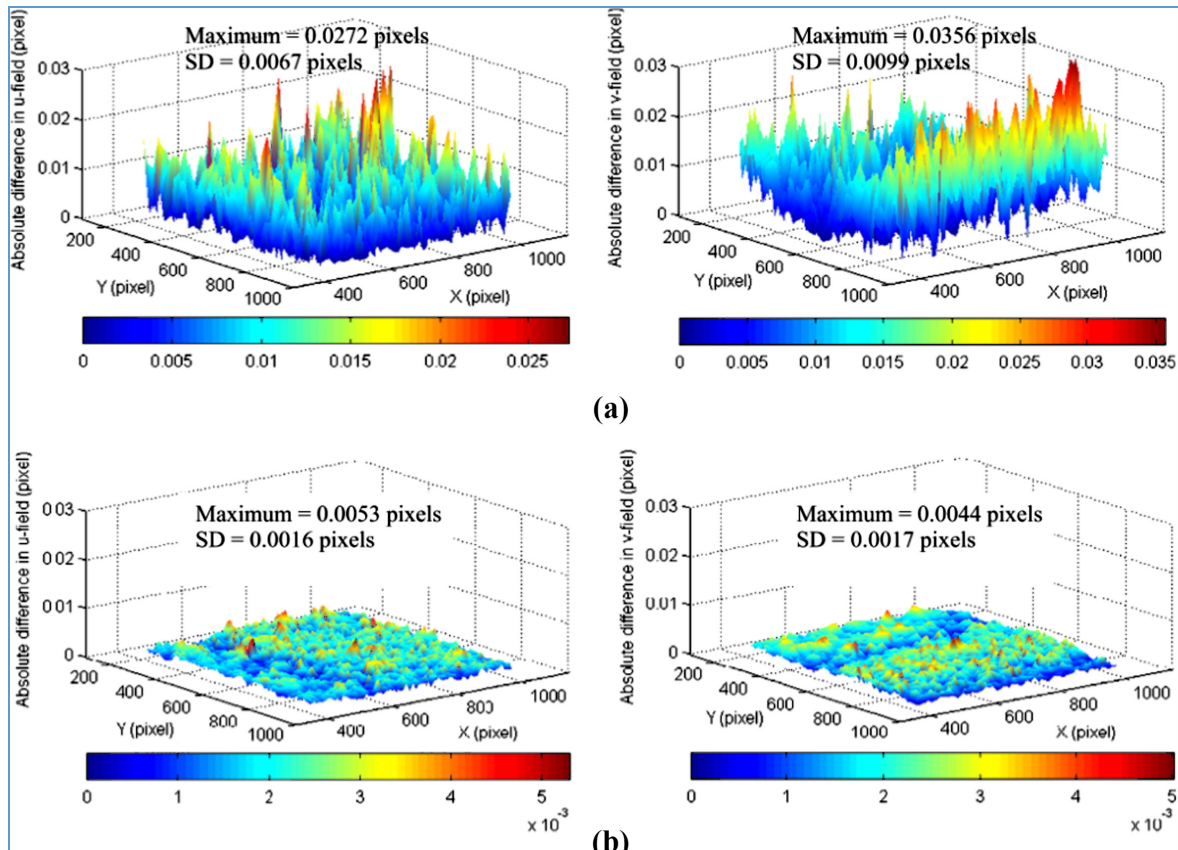

(a)

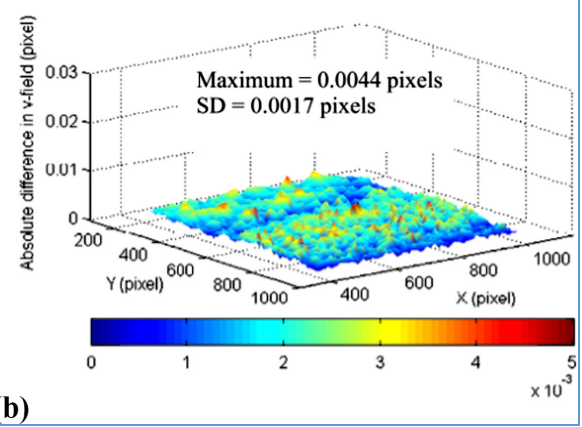

Book review

Figure 5.

(a) Absolute difference in $u$-displacement (left) and $v$-displacement (right) fields measured by NR algorithm using quintic B-spline interpolation without Gaussian pre-filtering and quintic B-spline interpolation with

Gaussian pre-filtering and (b) absolute difference in $u$ displacement (left) and $v$-displacement (right) measured by NR algorithm using bicubic interpolation with Gaussian prefiltering and quintic Bspline interpolation with Gaussian prefiltering (Pan, 2013) 


\section{JIMSE 2,2}

\section{0}

Figure 6.

(a) Schematic representation and (b) top view of the actual imaging of experimental setup (Davis et al., 2018b); (c) the resultant surface textures after specimens were blasted with glass micro-beads having diameters of 50$100 \mu \mathrm{m}$ under different blasting pressures (Baizeau et al., 2017) and (d) the shear strain rate distribution for the five stages of deformation during the formation of a single saw-tooth segment at a cutting speed of $0.5 \mathrm{~m} / \mathrm{s}$ (Davis et al., 2018b) (a)

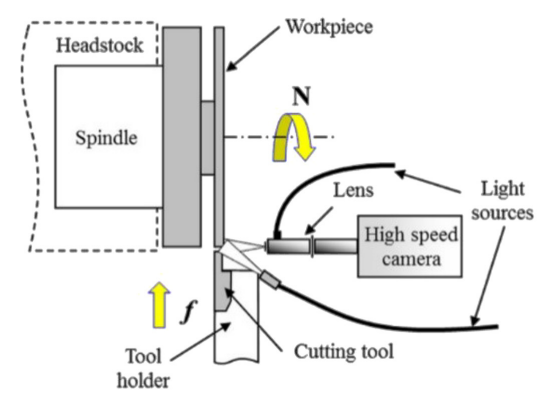

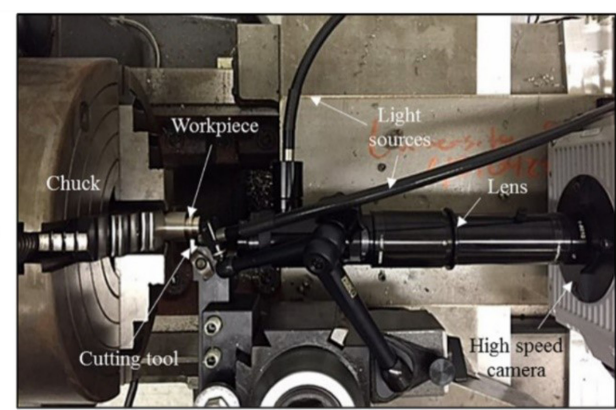

(b)

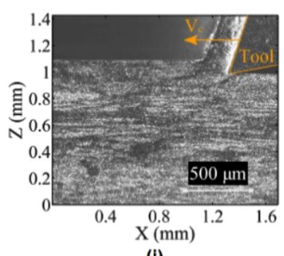

(i)

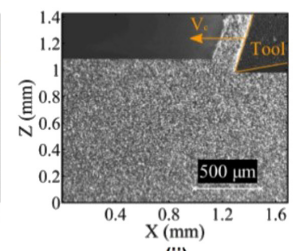

(ii)

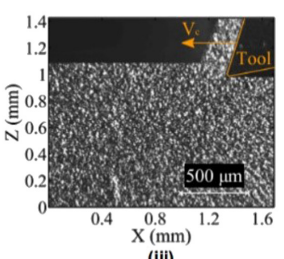

(iii) (c)

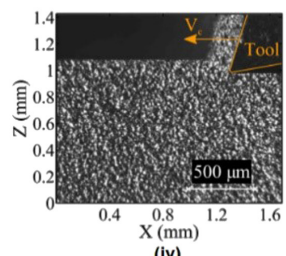

(iv)
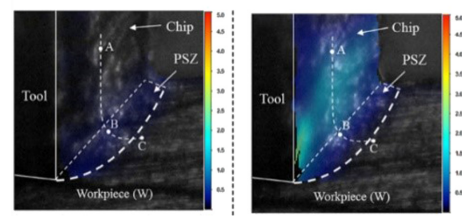

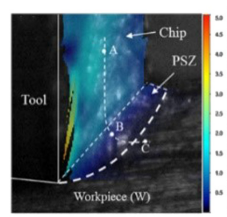

(d)
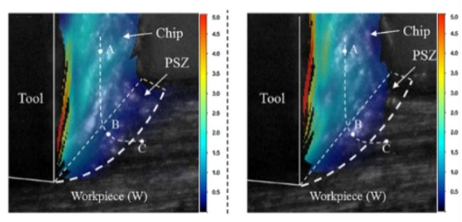

reach the order of one microsecond, and thus, the cutting process with a relatively high speed could be photographed. For instance, Baizeau et al. applied DIC with a double-frame camera to measure cutting forces at camera acquisition frequency. Subsequently, based on measurement results, the change of cutting forces is investigated when chip segments are formed (Baizeau et al., 2016). Further, Baizeau et al. used a high-speed double-frame imaging device with pulsed laser lighting to obtain sharp and highly resolved images during orthogonal cutting tests performed in an aluminum alloy in which the kinematic fields engendered by the cutting process are determined. The resultant surface textures after specimens were blasted with glass micro-beads having diameters of 50-100 $\mu \mathrm{m}$ under different blasting pressures are shown in Figure 6(c) (Baizeau et al., 2017). Despite the doubleframe cameras are well applied in high-speed cutting, they can only take two consecutive pictures, which makes it difficult to analyze the process effectively.

In general, compared with the traditional measurement method, the DIC technique is more suitable for the measurement during the cutting process because the cutting behavior has the features of small occurrence, severe deformation, short cutting process time, etc. The ability of DIC that could measure the chip formation process, including the intermediate quantity (e.g. strain and strain rate) in the cutting process, makes it possible to improve the quality of the workpiece, optimize the parameters and guide the production in the cutting process. Although the DIC technique offers some insight into the chip-formation mechanism, difficulties arise in identifying key features for high-speed cutting. Besides, the DIC technique 
is unsuitable for the estimation of shear strain and shear strain rate when the sliding during chip formation is not a continuous deformation process, i.e., brittle machining.

Welding: In the field of welding, DIC is mainly used in the analysis of the welded joint, including (1) uniaxial tensile measurement of welded joints for the study of constitutive behavior; (2) analysis of crack strain field of welded joint for studying fracture behavior; (3) analysis of welding thermal deformation field to study the residual stress evolution of the joint, etc.

The application of DIC technique to the study of the constitutive relationship of welded joints began in 1999; Reynolds and Duvall presented a series of tensile tests of aluminum alloy specimens that are spliced by friction welding (Reynolds and Duvall, 1999). This work has been shown that if the property gradients in a weld are not too steep, then the constitutive properties of the micro-structural regions within a weld can be deduced from a single tensile test coupled with the DIC full-field displacement technique. Subsequently, Genevois et al. investigated the effect of the microstructure heterogeneity on the global and local tensile properties of friction-stir-welded joints in aluminum alloys via the DIC technique (Genevois et al., 2006). Lockwood et al. examined the global and local mechanical response of frictionstir-welded AA2024 experimentally and numerically and full-field strain measurements were obtained on transversely loaded tensile specimens via the DIC technique. The microstructure corresponding to the 2024 friction stir welding is shown in Figure 7(c) (Lockwood et al., 2002). Leitão et al. presented the characterization of the constitutive behavior of friction stir welds based on the DIC technique in which the constitutive behavior of different weld regions was assessed from local tensile stress-strain curves. Transverse tensile specimen, strain maps, stress-strain curves and hardness profile for the S55 weld are shown in Figure 7(d) and (e) (Leitão et al., 2012).

Some researchers have also applied the DIC technique in fatigue tests, such as the crack growth analysis in welded joints. For instance, Chen et al. proposed the strategy that using the

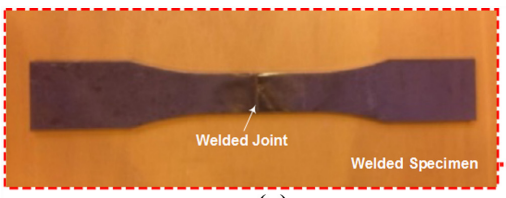

(a)

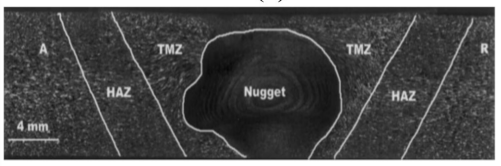

(c)

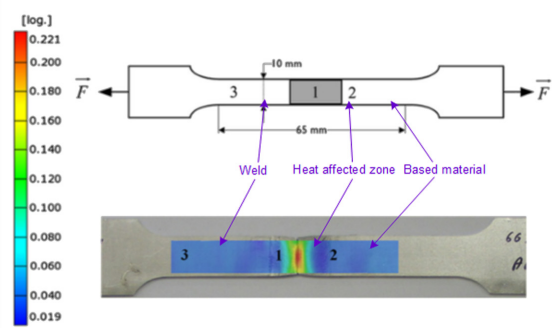

(d)

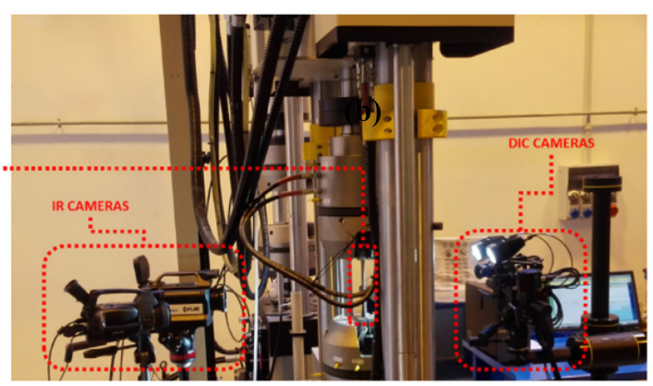

(b)

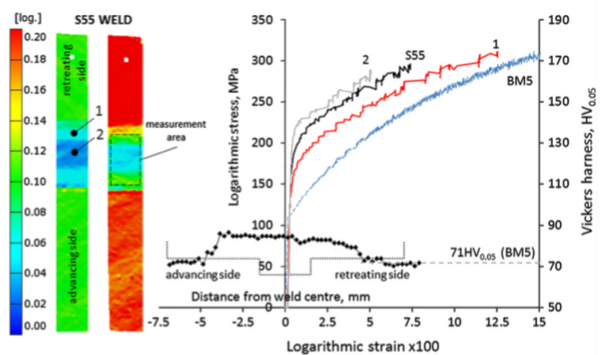

(e)
Book review

51

Figure 7.

(a) Welded specimen and (b) experimental setup of the measurement system (Corigliano et al., 2017); (c) the microstructure corresponding to the friction stir welding, where thermo-

mechanically affected zone (TMZ), heat

affected zone (HAZ) and fine grain

recrystallized zone (nugget) are presented and the welding

direction is into the page and the advancing and

retreating sides are labeled $\mathrm{A}$ and $\mathrm{R}$, respectively

(Lockwood et al., 2002);

(d) Schematic of a fullsize transverse tensile specimen (upper) and an image of the major logarithmic strain distribution after maximum load, acquired using DIC, in a tensile sample and (e)

Strain maps, stressstrain curves and hardness profile for the S55 weld (Leitão et al., 2012) 
JIMSE

2,2

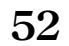

DIC technique coupled with thermo-elastic stress analysis to monitor the crack propagation process of T-joint specimens during the fatigue test (Chen et al., 2018). Corigliano et al. carried out static tensile and fatigue tests on butt-welded specimens made of S355 steel in which two full-field techniques were applied during the tests: DIC and infrared thermography techniques (Corigliano et al., 2017). The welded specimen and experimental setup are shown in Figure 7(a) and (b), respectively.

The DIC technique has also been employed to study the residual stress evolution of the welded joint. $\mathrm{Xu}$ et al. presented an incremental cutting method for evaluating the longitudinal residual stresses in the butt-welded thin plate by employing the DIC measurement technique (Xu and Bao, 2017). Kubit analyzed the strain distribution and residual stress of joints welded with the friction-stir-welding method after shot peening treatment in which the three-dimensional DIC method is used (Kubit et al., 2020). Generally, the DIC technique not only overcomes the problem that the deformation of the hightemperature weld area cannot be measured in real time, but also provides a validation method for numerical simulation theory. Although DIC methods have great advantages over the conventional measurement methods (e.g. heat-resisting strain gage) in the welding process, some challenges still remain for delivering the full potential in the welding field, including the effect of intense arc, the ablation of the speckle pattern owing to high temperature, oxidation of weldment surface interferes with image matching, etc.

Forming: In recent years, the application of the DIC technique has become increasingly popular in the forming process because it allows automatic evaluation of strains without tedious measurement of circle grids (Silva et al., 2015). Especially for the experimental characterization of the formability limits, the DIC technique has the additional advantage of avoiding manual intervention that usually leads to undesired measurement errors. In most cases, DIC is applied in the research of sheet metal forming for determining the plastic strain ratio of sheet metal and obtaining a reliable forming limit diagram (FLD) that both are important tools to evaluate the workability of metal sheets. For instance, Huang et al. determined the forming limit curve that is a key component in an FLD of sheet metal by using the DIC technique (Huang and Shi, 2017). Decultot $e t$ al. reported the development of strain fields encountered in incremental forming and evaluated the material formability on several formed shapes in single point incremental forming that is a promising sheet metal forming process for prototyping and small batches (Decultot et al., 2010). In order to obtain a more accurate FLD of the specimen, it is necessary to analyze the strain path during the deformation process. Besides, Mecedes et al. examined the geometric accuracy and thickness distributions using the DIC technique for studying the performance of multistage incremental sheet forming in formability, accuracy and uniform level of sheet thickness. The experimental setup, the image of the form and the corresponding 3D thickness model based on DIC data are shown in Figure 8(a-c), respectively (Mercedes et al., 2019). Wang et al. compared several different DIC procedures to establish an FLD and proposed a DIC-based time-dependent measurement method for the forming limit strains. This procedure is considered as more accurately defining the onset of necking and the boundary of safe deformation of an FLD as shown in Figure 8(e) (Wang et al., 2014). Compared with the conventional strain gage measurement that could only acquire the strain of fractural moment, the DIC method could measure the strain in the whole deformation process of any point in the specimen. For example, Cramer et al. quantified forming limits and retained austenite transformation in Q\&P 1080 steel as a function of plastic strain levels for three different strain paths as shown in Figure 8(f) (Cramer et al., 2018).

Besides, the DIC technique has been also used in bulk metal forming, but the utilization has been limited to the determination of the stress-strain curves. Magrinho et al. studied the formability limits by fracture obtained from standard bulk metal forming tests performed with cylindrical, tapered and flanged specimens in which DIC was used to determine strain 


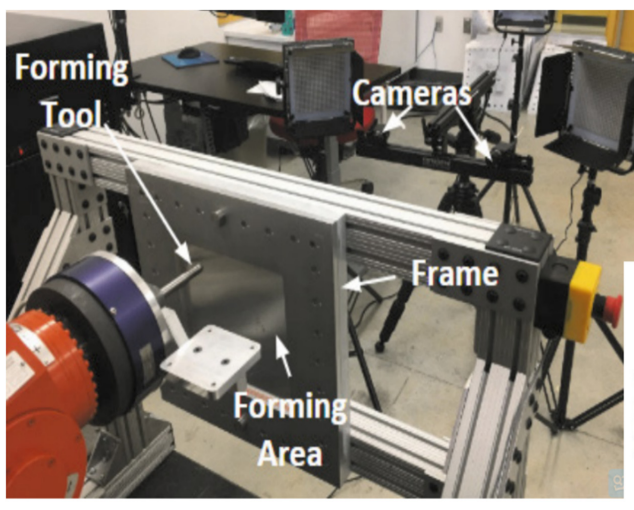

(a)

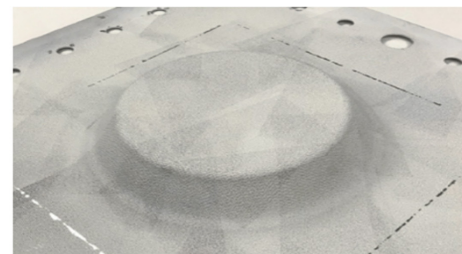

(b)

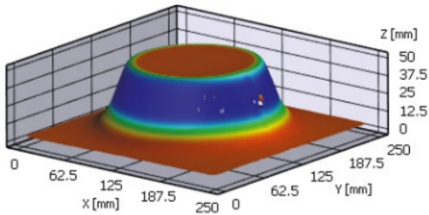

(c)

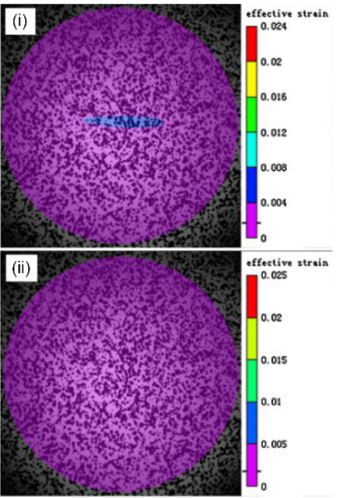

(e)

(d)

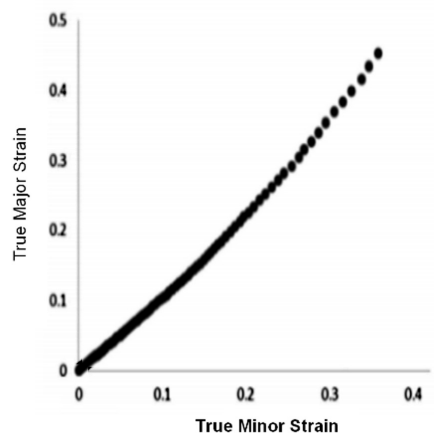

(i)
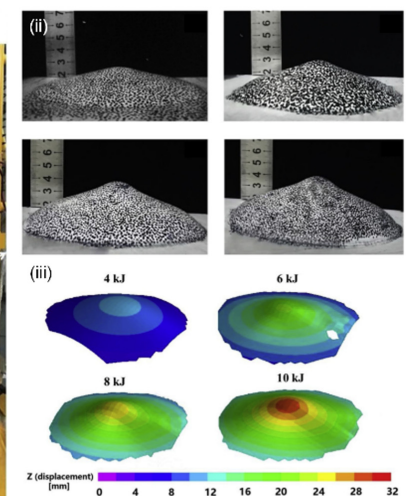

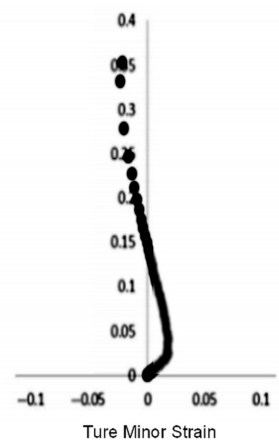

(ii)

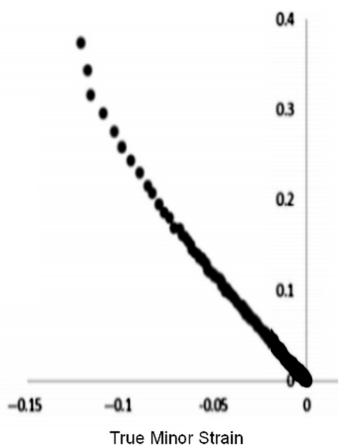

(iii)

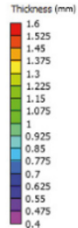
of multi-stage incremental sheet forming using DIC; (b) image of form and (c) the corresponding $3 \mathrm{D}$ thickness model based on DIC data (Mercedes et al., 2019); (d) EMF system with DIC: (i) an overview of EMF experimental arrangement, (ii) the contour of post-test which were freeformed at $4 \mathrm{~kJ}, 6 \mathrm{~kJ}, 8 \mathrm{~kJ}$, $10 \mathrm{~kJ}$ and (iii) the dome height contour measured by the DIC at corresponding energy (Deng et al., 2019); (e) effect of the gradient of the strain increments on the determination of the onset of localized necking: image with the gradient of (i) 0.004 and (ii) 0.005 by using DIC technique (Wang et al., 2014) and (f) strain paths from DIC measurements for (i) biaxial tension specimen, (ii) planestrain tension specimen and (iii) uniaxial tension specimen (Cramer et al., 2018)

(f)

paths and immersion of steel specimens (Magrinho et al., 2018). Except for sheet and bulk metal forming, the application of the DIC technique in tube forming has also been reported. Cristino et al. made use of DIC technique, thickness measurements and force-displacement 
JIMSE 2,2

evolutions to obtain the strain loading paths and the strain values at the onsets of failure by necking and fracture in tube forming (Cristino et al., 2019). Additionally, DIC technology has been used in some advanced forming processes, such as electromagnetic forming (EMF) that can improve the forming limit under room temperature. Deng $e$ t al. adopted DIC technology to calculate the full-field displacement, velocity, strain and strain rate with two high-speed cameras in the EMF process as shown in Figure 8(d) (Deng et al., 2019).

Typically, the DIC method is non-contacting and able to complete the measurement of the strain data of a specimen within a few minutes in the forming process. Besides, the DIC technique can not only measure the strain of the final state of sheet metal forming, but also can quickly and intuitively measure the strain distribution of the specimen surface during the entire forming process. Also, it could overcome the many shortcomings of conventional measurement methods, such as difficulty to repeat positioning, complicated procedures, timeconsuming, unable to in situ measure, etc. Despite the rising popularity of the DIC, there remain some major technical challenges during the DIC data analysis for metal forming; for instance, it is hard to precisely pinpoint strains at the onset of localized necking.

Additive manufacturing: With the ever-growing demands of high-performance products with low lead times and more customized products, additive manufacturing has attracted more and more attention. However, in the manufacturing process, components are usually accompanied by some flaws such as shape deviations, cracking, and residual stresses, due to rapid heating, solidification, etc., which directly affects the mechanical performance of the components. In order to improve or avoiding the flaws mentioned above, researchers try to glean the mechanical properties of printed specimens via accurate measurement and then use these data as a basis to guide the optimization of the parameters in the manufacturing process. As a remarkable measurement method that takes advantage of robust, wide dynamic range and is not affected by rigid body motions and ambient vibrations, the DIC technique has been widely applied into advanced additive manufacturing for obtaining displacements and strains of printed components. Biegler $e t$ al. employed the DIC technique along with optical filters to measure in situ distortions on a wall geometry produced with laser metal deposition (LMD), experimental setup and in situ images are shown in Figure 9(a-c) (Biegler et al., 2018). In this work, the suitability of the DIC technique for measurements on an AM sample is shown and the potential for the validated numerical model as a predictive tool to reduce trial-and-error and improve part quality is evaluated. Ocelík et al. measured the in situ displacements and strains occurring on the substrate underside during a single laser metal deposition path, which provides very useful information concerning laser surface treatment and laser cladding that could help to optimize the laser cladding parameters and suppress the formation of high-level internal strains (Ocelík et al., 2009). Wu et al. explored the ability of DIC used in conjunction with sectioning to measure deformations occurring in selective laser melting (SLM) samples due to the removal from the substrate plate after processing (Wu et al., 2014). Additionally, as advanced non-contact instrumentation, DIC does not depend on the size of the specimen, which provides greater versatility for large additively manufactured components. For instance, Schnittker et al. verified the feasibility of using the DIC technique as a key enabler for robust data collection of strain measurements of large 3D printed components (Schnittker et al., 2019). Notably, DIC measurement combined with the finite element method (FEM) simulation could provide experimental support for the mechanical feasibility of a 3D printed specimen. For instance, Sutradhar et al. examined the feasibility of implants designed by the topology optimization method for craniofacial reconstructive surgery using DIC and FEM; the experimental setup and strain distribution comparison between DIC and FEA are shown in Figure 9(d) and (e) (Sutradhar et al., 2014). In general, DIC could capture full-field strain data with a good resolution on the surface of the model with complex geometries and inhomogeneity from images in the additive manufacturing process. Besides, the DIC technique could also be used for data collection 
of strain measurements of large 3D-printed parts. Even though DIC is a powerful instrument for the study of additive manufacturing, it is hard to involve acquired information into the system which directly controls the process in a feedback loop due to a time delay caused by limited heat transfer speed.

\section{Conclusion and prospects}

After more than 30 years of development, DIC has been successfully used in a wide range of fields, providing robust and accurate measurements. In this review, the historical development of DIC, the advantages of DIC and its measurement construction have been systematically reviewed. Then, the basic principles have been elaborated in detail. The possible error sources of the DIC technique are also discussed and some suggestions are given for improving the measurement accuracy. So far, the DIC technique has been widely applied in the manufacturing area, including cutting, welding, forming, additive manufacturing, etc. due to its high performance.

With the ever-growing quality of industrial cameras and the development of relevant algorithms, the measurement accuracy of DIC has been constantly improved, and it has become one of the most versatile and powerful tools of deformation measurement in manufacturing. Although tremendous progress has been made, some challenges still exist when high-performance DIC measurements are required, especially for intelligent manufacturing. Specifically, these challenges mainly focus on the following aspects.

The measurement of a specimen with poor natural texture or without speckle pattern: On some occasions, it is not allowed or impossible to make artificial speckle patterns on the

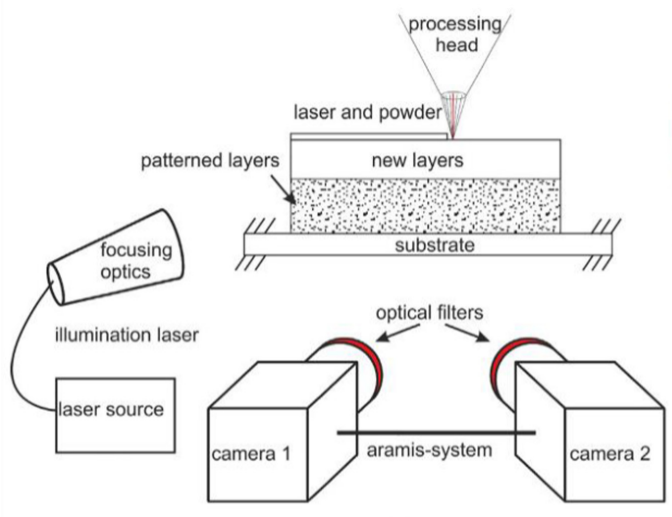

(a)

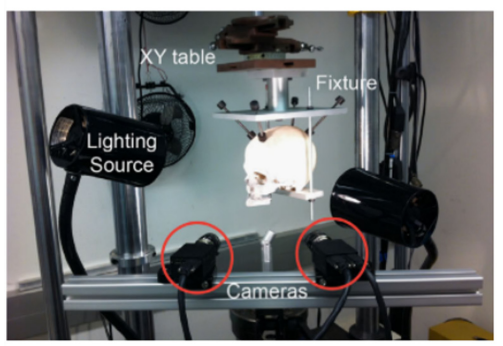

(d)

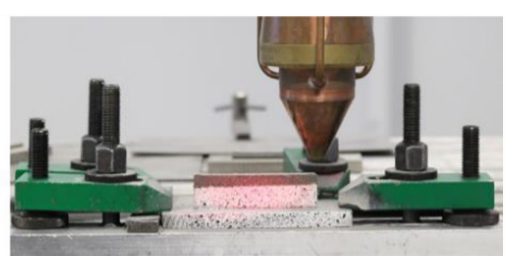

(b)

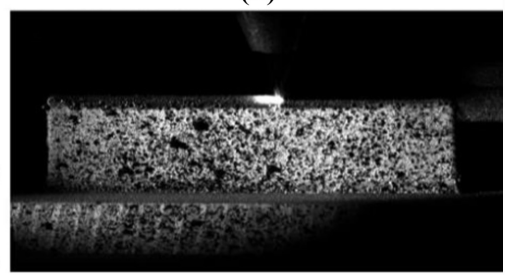

(c)

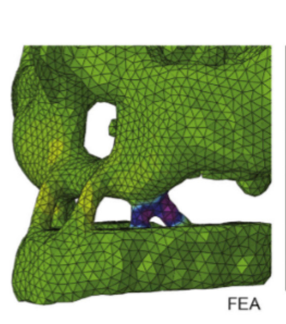

(e)
Figure 9.

(a) Schematic experimental setup for acquiring images of the laser-illuminated and patterned sample during LMD; (b) photograph of the setup after welding; (c) in situ image acquired by the left DIC camera in which patterned area is well visible even during welding, as the bright process is shielded by the optical filter (Biegler et al., 2018); (d) the configuration of the mechanical testing and data-capturing cameras and (e) vertical strain distribution comparison between FEA (left) and mechanical testing by DIC (right) (Sutradhar et al., 2014) 
JIMSE 2,2

surface of the specimen, which leads to the infeasibility of measurement using DIC, because there is a lack of sufficient texture information to support accurate tracking. Therefore, solving the measurement of specimens with low natural texture could greatly improve the application range of DIC.

The measurement of the kinematic field tightly coupled with the thermal field at high temperatures: During the manufacturing process, it is accompanied by evitable changes in the temperature field, especially for mechanical testing at high temperatures and material processing at high temperatures. Therefore, it is important to measure the kinematic field coupled with the thermal field of the specimen for quality control and parameter optimization in the manufacturing process at high temperatures. Although many researchers have made great efforts on this topic and many measurement methods are proposed for obtaining both kinematic and thermal fields, such as using two different imaging systems to observe two different surfaces of the specimen (Srinivasan et al., 2012) and using two imaging systems to observe the kinematic and thermal fields on the same specimen surface (Bodelot et al., 2009), however, there are some problems with these methods, including the inconsistency of space and time coordinates in the two imaging devices and the high cost and instability caused by the combination of two imaging devices, and the surface of the specimen cannot simultaneously satisfy the measurement of the two fields. Taking into account the above disadvantages, a few researchers tried to obtain simultaneously kinematic and thermal fields using a single type of camera (Maynadier et al., 2012; Orteu et al., 2008). Although preliminary exploration has been done, the problem that the DIC and infrared thermography have conflicting requirements for the specimen surface has not been addressed (Zhang et al., 2019a, b).

Adaptively select the subset size and shape function on different occasions: As mentioned before, on the one hand, the subset size should be big enough so that there is a sufficiently distinctive intensity pattern to support the reliable analysis of correlation; on the other hand, the corresponding shape function should accurately approximate local deformation within the target subset in order to reduce the systematic errors caused by under-matched shape functions or random errors caused by over-matched shape functions. There is still a lack of the mechanism of adaptively selecting the subset and shape function, since the actual deformation occurring in each subset cannot be obtained in advance. Besides, in intelligent manufacturing with high requirements for intelligence and automation, the measurement technique needs to be sufficiently intelligent and automated to cope with different occasions. Thus, the adaptive selection of optimal subset size and shape function for subset matching is important for the application of the DIC technique in intelligent manufacturing.

The integration with modern design and simulation tools: As mentioned above, intelligent manufacturing imposes a high requirement in automation. The combination of DIC with computer-aided design (CAD) technology and FEM could provide the designing scheme, feasibility analysis and accurate measurement of products at once, which will greatly improve the automation and efficiency of intelligent manufacturing systems.

\section{References}

Asseban, A., et al. (2000), "Digital speckle photography and speckle tomography in heat transfer studies", Optics and Laser Technology, Vol. 32 Nos 7-8, pp. 583-592, doi: 10.1016/S0030-3992(00) 00095-5.

Audrey, G.Z., Robert, W. and Davidson, R.B.H. (1983), "Strain measurement in wood using a digital image correlation technique", Wood and Fiber Science, Vol. 27 No. 4, pp. 346-359, available at: https://wfs.swst.org/index.php/wfs/article/view/1391/1391.

Baizeau, T., et al. (2016), "Cutting force sensor based on digital image correlation for segmented chip formation analysis", Journal of Materials Processing Technology, Vol. 238, pp. 466-473, doi: 10. 1016/j.jmatprotec.2016.07.016. 
Baizeau, T., et al. (2017), "Kinematic field measurements during orthogonal cutting tests via DIC with double-frame camera and pulsed laser lighting', experimental mechanics", Experimental Mechanics, Vol. 57 No. 4, pp. 581-591, doi: 10.1007/s11340-016-0248-9.

Bastawros, A.F., Bart-Smith, H. and Evans, A.G. (2000), "Experimental analysis of deformation mechanisms in a closed-cell aluminum alloy foam", Journal of the Mechanics and Physics of Solids, Vol. 48 No. 2, pp. 301-322, doi: 10.1016/S0022-5096(99)00035-6.

Berfield, T.A., et al. (2006), "Fluorescent image correlation for nanoscale deformation measurements", Small, Vol. 2 No. 5, pp. 631-635, doi: 10.1002/smll.200500289.

Besnard, G., Hild, F. and Roux, S. (2006), "Finite-element' displacement fields analysis from digital images: application to Portevin-Le Châtelier bands", Experimental Mechanics, Vol. 46 No. 6, pp. 789-803, doi: 10.1007/s11340-006-9824-8.

Biegler, M., Graf, B. and Rethmeier, M. (2018), "In-situ distortions in LMD additive manufacturing walls can be measured with digital image correlation and predicted using numerical simulations", Additive Manufacturing, Elsevier B.V, Vol. 20, pp. 101-110, doi: 10.1016/j. addma.2017.12.007.

Bodelot, L., et al. (2009), "Experimental setup for fully coupled kinematic and thermal measurements at the microstructure scale of an AISI 316L steel", Materials Science and Engineering A, Vol. 501 Nos 1-2, pp. 52-60, doi: 10.1016/j.msea.2008.09.053.

Bornert, M., et al. (2009), "Assessment of digital image correlation measurement errors: methodology and results", Experimental Mechanics, Vol. 49 No. 3, pp. 353-370, doi: 10.1007/s11340-0089204-7.

Bruck, H.A., et al. (1989), "Digital image correlation using Newton-Raphson method of partial differential correction”, Experimental Mechanics, Vol. 29 No. 3, pp. 261-267, doi: 10.1007/ BF02321405.

Budyn, E., Jonvaux, J. and Hoc, T. (2012), "Digital image correlation of bone sequential microscopic observations", International Journal for Numerical Methods in Biomedical Engineering, Vol. 28 No. 8, pp. 815-837, doi: 10.1002/cnm.1492.

Calvié, E., et al. (2014), "Mechanical behavior law of ceramic nanoparticles from transmission electron microscopy in situ nano-compression tests", Materials Letters, Elsevier, Vol. 119, pp. 107-110, doi: 10.1016/j.matlet.2014.01.002.

Chang, S., et al. (2005), "Nanoscale in-plane displacement evaluation by AFM scanning and digital image correlation processing", Nanotechnology, Vol. 16 No. 4, pp. 344-349, doi: 10.1088/09574484/16/4/002.

Chen, D., et al. (2018), "Crack growth analysis in welded and non-welded T-joints based on lock-in digital image correlation and thermoelastic stress analysis", International Journal of Fatigue, Elsevier, Vol. 110 November, pp. 172-185, doi: 10.1016/j.ijfatigue.2018.01.020.

Chevalier, L., et al. (2001), "Digital image correlation used to analyze the multiaxial behavior of rubberlike materials", European Journal of Mechanics, A/Solids, Vol. 20 No. 2, pp. 169-187, doi: 10.1016/ S0997-7538(00)01135-9.

Cho, S., Cárdenas-García, J.F. and Chasiotis, I. (2005), "Measurement of nanodisplacements and elastic properties of MEMS via the microscopic hole method", Sensors and Actuators, A: Physical, Vol. 120 No. 1, pp. 163-171, doi: 10.1016/j.sna.2004.11.028.

Choi, S. and Shah, S.P. (1997), "Measurement of deformations on concrete subjected to compression using image correlation", Experimental Mechanics, Vol. 37 No. 3, pp. 307-313, doi: 10.1007/ BF02317423.

Chu, T.P. and Poudel, A. (2014), "Digital image correlation techniques for aerospace applications", ASNT Fall Conference, No. 618, p. 9.

Corigliano, P., et al. (2017), "Fatigue analysis of marine welded joints by means of DIC and IR images during static and fatigue tests", Engineering Fracture Mechanics, Elsevier, Vol. 183, pp. 26-38, doi: 10.1016/j.engfracmech.2017.06.012. 
JIMSE 2,2

Cramer, J., et al. (2018), "Effect of strain path on forming limits and retained austenite transformation in Q\&P 1180 steel”, Materials Science and Engineering A, Vol. 734 July, pp. 192-199, doi: 10. 1016/j.msea.2018.07.062.

Cristino, V.A.M., et al. (2019), "A digital image correlation based methodology to characterize formability in tube forming", Journal of Strain Analysis for Engineering Design, Vol. 54 No. 2, pp. 139-148, doi: 10.1177/0309324718823629.

Davis, B., Dabrow, D., Newell, R., et al. (2018a), "Chip morphology and chip formation mechanisms during machining of ECAE-processed titanium", Journal of Manufacturing Science and Engineering, Transactions of the ASME, Vol. 140 No. 3, pp. 1-12, doi: 10.1115/1.4038442.

Davis, B., Dabrow, D., Ifju, P., et al. (2018b), "Study of the shear strain and shear strain rate progression during titanium machining", Journal of Manufacturing Science and Engineering, Transactions of the ASME, Vol. 140 No. 5, pp. 1-13, doi: 10.1115/1.4038891.

Decultot, N., et al. (2010), "Single point incremental sheet forming investigated by in-process 3D digital image correlation”, EPJ Web of Conferences, Vol. 6, pp. 1-8, doi: 10.1051/epjconf/20100611001.

Deng, H., et al. (2019), "A study of electromagnetic free forming in AA5052 using digital image correlation method and FE analysis", Journal of Manufacturing Processes, Vol. 37 January, pp. 595-605, doi: 10.1016/j.jmapro.2018.12.033.

Dong, Y.L. and Pan, B. (2017), "A review of speckle pattern fabrication and assessment for digital image correlation”, Experimental Mechanics, Experimental Mechanics, Vol. 57 No. 8, pp. 1161-1181, doi: 10.1007/s11340-017-0283-1.

Franck, C., et al. (2007), "Three-dimensional full-field measurements of large deformations in soft materials using confocal microscopy and digital volume correlation”, Experimental Mechanics, Vol. 47 No. 3, pp. 427-438, doi: 10.1007/s11340-007-9037-9.

Genevois, C., Deschamps, A. and Vacher, P. (2006), "Comparative study on local and global mechanical properties of 2024 T351, 2024 T6 and 5251 O friction stir welds", Materials Science and Engineering A, Vol. 415 Nos 1-2, pp. 162-170, doi: 10.1016/j.msea.2005.09.032.

Georges, M. (2019), Holographic Interferometry: from History to Modern Applications, Optical Holography: Materials, Theory and Applications, Elsevier, doi: 10.1016/B978-0-12-815467-0.00006-2.

Grant, I. (1997), "Particle image velocimetry: a review", Proceedings of the Institution of Mechanical Engineers, Part C: Journal of Mechanical Engineering Science, Vol. 211 No. 1, pp. 55-76, doi: 10. $1243 / 0954406971521665$.

Haddadi, H. and Belhabib, S. (2008), "Use of rigid-body motion for the investigation and estimation of the measurement errors related to digital image correlation technique", Optics and Lasers in Engineering, Vol. 46 No. 2, pp. 185-196, doi: 10.1016/j.optlaseng.2007.05.008.

Harangus, K. and Kakucs, A. (2014), "Finite-difference solution using displacement potential function for plane stresses and displacements", Procedia Technology, Vol. 12, pp. 394-400, doi: 10.1016/j. protcy.2013.12.505.

He, Z.H., et al. (1984), "Two-dimensional fluid-velocity measurements by use of digital-speckle correlation techniques", Experimental Mechanics, Vol. 24 No. 2, pp. 117-121, doi: 10.1007/ BF02324993.

Helm, J.D. (1996), "Improved three-dimensional image correlation for surface displacement measurement", Optical Engineering, Vol. 35 No. 7, p. 1911, doi: 10.1117/1.600624.

Hild, F. and Roux, S. (2006), "Digital image correlation: from displacement measurement to identification of elastic properties - a review", Strain, Vol. 42 No. 2, pp. 69-80, doi: 10.1111/j.14751305.2006.00258.x.

Hild, F. and Roux, S. (2012), "Comparison of local and global approaches to digital image correlation", Experimental Mechanics, Vol. 52 No. 9, pp. 1503-1519, doi: 10.1007/s11340-012-9603-7.

Huang, L. and Shi, M. (2017), "Determination of the forming limit curve using digital image correlation - comparison of different approaches to pinpoint the onset of localized necking", SAE Technical Papers, 2017-March, doi: 10.4271/2017-01-0301. 
Jin, H. (2005), “Theoretical development for pointwise digital image correlation”, Optical Engineering, Vol. 44 No. 6, p. 067003, doi: 10.1117/1.1928908.

Jin, H. and Bruck, H.A. (2005), "A new method for characterizing nonlinearity in scanning probe microscopes using digital image correlation”, Nanotechnology, Vol. 16 No. 9, pp. 1849-1855, doi: $10.1088 / 0957-4484 / 16 / 9 / 072$.

Kang, J., et al. (2005), "Microscopic strain mapping using scanning electron microscopy topography image correlation at large strain", The Journal of Strain Analysis for Engineering Design, Vol. 40 No. 6, pp. 559-570, doi: 10.1243/030932405X16151.

Knauss, W.G., Chasiotis, I. and Huang, Y. (2003), "Mechanical measurements at the micron and nanometer scales", Mechanics of Materials, Vol. 35 Nos 3-6, pp. 217-231, doi: 10.1016/S01676636(02)00271-5.

Kubit, A., et al. (2020), "Application of the 3D digital image correlation to the analysis of deformation of joints welded with the FSW method after shot peening", Advances in Materials Science, Vol. 19 No. 4, pp. 57-66, doi: 10.2478/adms-2019-0022.

Lecompte, D., et al. (2006), "Quality assessment of speckle patterns for digital image correlation", Optics and Lasers in Engineering, Vol. 44 No. 11, pp. 1132-1145, doi: 10.1016/j.optlaseng.2005. 10.004 .

Leitão, C., et al. (2012), "Determination of local constitutive properties of aluminium friction stir welds using digital image correlation”, Materials and Design, Vol. 33 No. 1, pp. 69-74, doi: 10.1016/j. matdes.2011.07.009.

Leprince, S., et al. (2007), "Automatic and precise orthorectification, coregistration, and subpixel correlation of satellite images, application to ground deformation measurements", IEEE Transactions on Geoscience and Remote Sensing, Vol. 45 No. 6, pp. 1529-1558, doi: 10.1109/ TGRS.2006.888937.

Little, E.G. (1992), "Strain gauge measurement", Strain Measurement in Biomechanics, Springer, Dordrecht, pp. 39-57, doi: 10.1007/978-94-011-2330-3_3.

Lockwood, W.D., Tomaz, B. and Reynolds, A.P. (2002), "Mechanical response of friction stir welded AA2024: experiment and modeling", Materials Science and Engineering A, Vol. 323 Nos 1-2, pp. 348-353, doi: 10.1016/S0921-5093(01)01385-5.

Louis, L., Wong, T.F. and Baud, P. (2007), "Imaging strain localization by X-ray radiography and digital image correlation: deformation bands in Rothbach sandstone", Journal of Structural Geology, Vol. 29 No. 1, pp. 129-140, doi: 10.1016/j.jsg.2006.07.015.

Luo, M. (2006), "Displacement/strain measurements using an optical microscope and digital image correlation", Optical Engineering, Vol. 45 No. 3, p. 033605, doi: 10.1117/1.2182108.

Magrinho, J.P., et al. (2018), "New methodology for the characterization of failure by fracture in bulk forming", Journal of Strain Analysis for Engineering Design, Vol. 53 No. 4, pp. 242-247, doi: 10. 1177/0309324718758842.

Maynadier, A., et al. (2012), "One-shot measurement of thermal and kinematic fields: InfraRed image correlation (IRIC)”, Experimental Mechanics, Vol. 52 No. 3, pp. 241-255, doi: 10.1007/s11340-0119483-2.

Mercedes, M.G., et al. (2019), "Analysis of geometric accuracy and thickness reduction in multistage incremental sheet forming using digital image correlation", Procedia Manufacturing, Elsevier B.V, Vol. 34, pp. 950-960, doi: 10.1016/j.promfg.2019.06.105.

Niu, Y., et al. (2018), “A comprehensive solution for electronic packages' reliability assessment with digital image correlation (DIC) method", Microelectronics Reliability, Elsevier, Vol. 87 January, pp. 81-88, doi: 10.1016/j.microrel.2018.06.006.

Ocelík, V., Bosgra, J. and de Hosson, J.T.M. (2009), "In-situ strain observation in high power laser cladding", Surface and Coatings Technology, Elsevier B.V, Vol. 203 Nos 20-21, pp. 3189-3196, doi: 10.1016/j.surfcoat.2009.03.050. 
JIMSE 2,2

60

Orteu, J.J., et al. (2008), "An innovative method for 3-D shape, strain and temperature full-field measurement using a single type of camera: principle and preliminary results", Experimental Mechanics, Vol. 48 No. 2, pp. 163-179, doi: 10.1007/s11340-007-9071-7.

Palanca, M., Tozzi, G. and Cristofolini, L. (2016), "The use of digital image correlation in the biomechanical area: a review", International Biomechanics, Taylor \& Francis, Vol. 3 No. 1, pp. 1-21, doi: 10.1080/23335432.2015.1117395.

Pan, B. (2009), "Reliability-guided digital image correlation for image deformation measurement", Applied Optics, Vol. 48 No. 8, pp. 1535-1542, doi: 10.1364/AO.48.001535.

Pan, B. (2013), "Bias error reduction of digital image correlation using Gaussian pre-filtering", Optics and Lasers in Engineering, Elsevier, Vol. 51 No. 10, pp. 1161-1167, doi: 10.1016/j.optlaseng.2013. 04.009.

Pan, B. (2018), "Digital image correlation for surface deformation measurement: historical developments, recent advances and future goals", Measurement Science and Technology, IOP Publishing, Vol. 29 No. 8, doi: 10.1088/1361-6501/aac55b.

Pan, B., et al. (2006), "Performance of sub-pixel registration algorithms in digital image correlation", Measurement Science and Technology, Vol. 17 No. 6, pp. 1615-1621, doi: 10.1088/0957-0233/17/ 6/045.

Pan, B., et al. (2008), "Study on subset size selection in digital image correlation for speckle patterns", Optics Express, Vol. 16 No. 10, p. 7037, doi: 10.1364/oe.16.007037.

Pan, B., et al. (2009), "Two-dimensional digital image correlation for in-plane displacement and strain measurement: a review", Measurement Science and Technology, Vol. 20 No. 6, doi: 10.1088/09570233/20/6/062001.

Pan, B., Yu, L.P. and Zhang, Q.B. (2018), "Review of single-camera stereo-digital image correlation techniques for full-field 3D shape and deformation measurement", Science China Technological Sciences, Vol. 61 No. 1, pp. 2-20, doi: 10.1007/s11431-017-9090-x.

Peters, W.H., et al. (1983), "Application of digital correlation methods to rigid body mechanics", Optical Engineering, Vol. 22 No. 6, doi: 10.1117/12.7973231.

Pitter, M., et al. (2002), "Focus errors and their correction in microscopic deformation analysis using correlation”, Optics Express, Vol. 10 No. 23, p. 1361, doi: 10.1364/oe.10.001361.

Ramos, T., et al. (2015), "2D and 3D digital image correlation in civil engineering - measurements in a masonry wall”, Procedia Engineering, Elsevier B.V, Vol. 114, pp. 215-222, doi: 10.1016/j.proeng. 2015.08.061.

Réthoré, J., et al. (2005), "Estimation of mixed-mode stress intensity factors using digital image correlation and an interaction integral", International Journal of Fracture, Vol. 132 No. 1, pp. 65-79, doi: 10.1007/s10704-004-8141-4.

Reu, P. (2014), “All about speckles: speckle size measurement”, Experimental Techniques, Vol. 38 No. 6, pp. 1-2, doi: 10.1111/ext.12110.

Reynolds, A.P. and Duvall, F. (1999), "Digital image correlation for determination of weld and base metal constitutive behavior", Welding Journal (Miami, Fla), Vol. 78 No. 10, pp. 355-s.

Sabaté, N., et al. (2006), "Measurement of residual stresses in micromachined structures in a microregion”, Applied Physics Letters, Vol. 88 No. 7, pp. 1-4, doi: 10.1063/1.2177357.

Schnittker, K., et al. (2019), "Integrating digital image correlation in mechanical testing for the materials characterization of big area additive manufacturing feedstock", Additive Manufacturing, Elsevier, Vol. 26 September, pp. 129-137, doi: 10.1016/j.addma.2018.12.016.

Schreier, H.W. (2000), "Systematic errors in digital image correlation caused by intensity interpolation”, Optical Engineering, Vol. 39 No. 11, p. 2915, doi: 10.1117/1.1314593.

Schreier, H.W. and Sutton, M.A. (2002), "Systematic errors in digital image correlation due to undermatched subset shape functions", Experimental Mechanics, Vol. 42 No. 3, pp. 303-310, doi: 10.1177/001448502321548391. 
Schreier, H.W., Garcia, D. and Sutton, M.A. (2004), "Advances in light microscope stereo vision", Experimental Mechanics, Vol. 44 No. 3, pp. 278-288, doi: 10.1177/0014485104041546.

Schreier, H., Orteu, J.J. and Sutton, M.A. (2009), Image Correlation for Shape, Motion and Deformation Measurements: Basic Concepts, Theory and Applications, Image Correlation for Shape, Motion and Deformation Measurements: Basic Concepts, Theory and Applications, doi: 10.1007/978-0387-78747-3.

Shao, X., et al. (2020), "Digital image correlation with improved efficiency by pixel selection", Applied Optics, Vol. 59 No. 11, p. 3389, doi: 10.1364/ao.387678.

Silva, M.B., et al. (2015), "Recent approaches for the determination of forming limits by necking and fracture in sheet metal forming", Procedia Engineering, Elsevier B.V, Vol. 132, pp. 342-349, doi: 10.1016/j.proeng.2015.12.504.

Srinivasan, N., Raghu, N. and Venkatraman, B. (2012), "Study on lüders deformation in welded mild steel using infrared thermography and digital image correlation", Advanced Materials Research, Vol. 585, pp. 82-86, doi: 10.4028/www.scientific.net/AMR.585.82.

Stoilov, G., Kavardzhikov, V. and Pashkouleva, D. (2012), "A comparative study of random patterns for digital image correlation", Journal of Theoretical and Applied Mechanics, Vol. 42 No. 2, pp. 55-66, doi: 10.2478/v10254-012-0008-x.

Sun, Y. and Pang, J.H.L. (2006), "AFM image reconstruction for deformation measurements by digital image correlation”, Nanotechnology, Vol. 17 No. 4, pp. 933-939, doi: 10.1088/0957-4484/17/4/016.

Sun, Z., Lyons, J.S. and McNeill, S.R. (1997), "Measuring microscopic deformations with digital image correlation", Optics and Lasers in Engineering, Vol. 27 No. 4, pp. 409-428, doi: 10.1016/S01438166(96)00041-3.

Sutradhar, A., et al. (2014), "Experimental validation of 3D printed patient-specific implants using digital image correlation and finite element analysis", Computers in Biology and Medicine, Elsevier, Vol. 52, pp. 8-17, doi: 10.1016/j.compbiomed.2014.06.002.

Sutton, M., et al. (1983), "Determination of displacements using an improved digital correlation method", Image and Vision Computing, Vol. 1 No. 3, pp. 133-139, doi: 10.1016/0262-8856(83) 90064-1.

Sutton, M., et al. (1986), "Application of an optimized digital correlation method to planar deformation analysis", Image and Vision Computing, Vol. 4 No. 3, pp. 143-150, doi: 10.1016/0262-8856(86) 90057-0.

Sutton, M.A., et al. (2008), "The effect of out-of-plane motion on 2D and 3D digital image correlation measurements", Optics and Lasers in Engineering, Vol. 46 No. 10, pp. 746-757, doi: 10.1016/j. optlaseng.2008.05.005.

Tong, W. (2005), "An evaluation of digital image correlation criteria for strain mapping applications", Strain, Vol. 41 No. 4, pp. 167-175, doi: 10.1111/j.1475-1305.2005.00227.x.

Vend Roux, G. and Knauss, W.G. (1998), "Submicron deformation field measurements: part 2. Improved digital image correlation”, Experimental Mechanics, Vol. 38 No. 2, pp. 86-92, doi: 10. 1007/BF02321649.

Vendroux, G. and Knauss, W.G. (1998), "Submicron deformation field measurements: part 1. Developing a digital scanning tunneling microscope”, Experimental Mechanics, Vol. 38 No. 1, pp. 18-23, doi: 10.1007/BF02321262.

Vendroux, G., Schmidt, N. and Knauss, W.G. (1998), "Submicron deformation field measurements: part 3. Demonstration of deformation determinations", Experimental Mechanics, Vol. 38 No. 3, pp. 154-160.

Walker, C.A. (1994), "A historical review of moiré interferometry”, Experimental Mechanics, Vol. 34 No. 4, pp. 281-299, doi: 10.1007/BF02325143.

Wang, H., et al. (2006), "Error analysis of digital speckle correlation method under scanning electron microscope", Experimental Techniques, Vol. 30 No. 2, pp. 42-45, doi: 10.1111/j.1747-1567.2006. 00021.x. 
JIMSE 2,2

62

Wang, Y.Q., et al. (2009), "Quantitative error assessment in pattern matching: effects of intensity pattern noise, interpolation, strain and image contrast on motion measurements", Strain, Vol. 45 No. 2, pp. 160-178, doi: 10.1111/j.1475-1305.2008.00592.x.

Wang, K., et al. (2014), "Measuring forming limit strains with digital image correlation analysis", Journal of Materials Processing Technology, Vol. 214 No. 5, pp. 1120-1130, doi: 10.1016/j. jmatprotec.2014.01.001.

Wu, A.S., et al. (2014), "An experimental investigation into additive manufacturing-induced residual stresses in 316L stainless steel", Metallurgical and Materials Transactions A: Physical Metallurgy and Materials Science, Vol. 45 No. 13, pp. 6260-6270, doi: 10.1007/s11661-014-2549-x.

$\mathrm{Xu}, \mathrm{Y}$. and Bao, R. (2017), "Residual stress determination in friction stir butt welded joints using a digital image correlation-aided slitting technique", Chinese Journal of Aeronautics. Chinese Society of Aeronautics and Astronautics, Vol. 30 No. 3, pp. 1258-1269, doi: 10.1016/j.cja.2016. 11.003

Yamaguchi, I. (1981a), "Related content A laser-speckle strain”, Journal of Physics E: Scientific Instruments, Vol. 14, pp. 1270-1273.

Yamaguchi, I. (1981b), "Speckle displacement and decorrelation in the diffraction and image fields for small object deformation", Optica Acta, Vol. 28 No. 10, pp. 1359-1376, doi: 10.1080/713820454.

Yaofeng, S. and Pang, J.H.L. (2007), "Study of optimal subset size in digital image correlation of speckle pattern images", Optics and Lasers in Engineering, Vol. 45 No. 9, pp. 967-974, doi: 10. 1016/j.optlaseng.2007.01.012.

Yoneyama, S. (2006), "Lens distortion correction for digital image correlation by measuring rigid body displacement", Optical Engineering, Vol. 45 No. 2, p. 023602, doi: 10.1117/1.2168411.

Zhang, C. (2019), Quantitative Kinematic and Thermal Full Felds Measurement, Université de Lyon.

Zhang, D., Eggleton, C.D. and Arola, D.D. (2002), "Evaluating the mechanical behavior of arterial tissue using digital image correlation”, Experimental Mechanics, Vol. 42 No. 4, pp. 409-416, doi: $10.1007 / \mathrm{bf} 02412146$.

Zhang, J., et al. (2018), "A novel method for repeatedly generating speckle patterns used in digital image correlation", Optics and Lasers in Engineering, Elsevier, Vol. 100 August, pp. 259-266, doi: 10.1016/j.optlaseng.2017.09.012.

Zhang, C., et al. (2019a), "An innovative technique for real-time adjusting exposure time of siliconbased camera to get stable gray level images with temperature evolution", Mechanical Systems and Signal Processing, Elsevier, Vol. 122, pp. 419-432, doi: 10.1016/j.ymssp.2018.12.042.

Zhang, C., et al. (2019b), "Application of silicon-based camera for measurement of non-homogeneous thermal field on realistic specimen surface", Applied Thermal Engineering, Vol. 149, pp. 1186-1191, doi: 10.1016/j.applthermaleng.2018.12.114.

Zhou, P. (2001), "Subpixel displacement and deformation gradient measurement using digital image/ speckle correlation (DISC)", Optical Engineering, Vol. 40 No. 8, p. 1613, doi: 10.1117/1.1387992.

Zhou, J., et al. (2018), "Toward new-generation intelligent manufacturing", Engineering. Chinese Academy of Engineering, Vol. 4 No. 1, pp. 11-20, doi: 10.1016/j.eng.2018.01.002.

\section{Corresponding author}

Jun Zou can be contacted at: junzou@zju.edu.cn

For instructions on how to order reprints of this article, please visit our website:

www.emeraldgrouppublishing.com/licensing/reprints.htm

Or contact us for further details: permissions@emeraldinsight.com 